\title{
MODEL PSEUDOCONVEX DOMAINS AND BUMPING
}

\author{
GAUTAM BHARALI
}

\begin{abstract}
The Levi geometry at weakly pseudoconvex boundary points of domains in $\mathbb{C}^{n}, n \geq 3$, is sufficiently complicated that there are no universal model domains with which to compare a general domain. Good models may be constructed by bumping outward a pseudoconvex, finite-type $\Omega \subset \mathbb{C}^{3}$ in such a way that: $i$ ) pseudoconvexity is preserved, $i i$ ) the (locally) larger domain has a simpler defining function, and $i i i)$ the lowest possible orders of contact of the bumped domain with $\partial \Omega$, at the site of the bumping, are realised. When $\Omega \subset \mathbb{C}^{n}, n \geq 3$, it is, in general, hard to meet the last two requirements. Such well-controlled bumping is possible when $\Omega$ is $h$-extendible/semiregular. We examine a family of domains in $\mathbb{C}^{3}$ that is strictly larger than the family of $h$-extendible/semiregular domains and construct explicit models for these domains by bumping.
\end{abstract}

\section{INTRODUCTION}

A rather successful strategy for understanding a pseudoconvex domain in $\mathbb{C}^{n}, n \geq$ 2 , involves carefully deforming its boundary about some boundary point without destroying pseudoconvexity so that the new domain "well approximates" the original but has a much simpler defining function. In many instances, one requires the model domain to be deformed outwards about the chosen boundary point. The latter procedure is formalised as follows:

(*) Given a smoothly bounded pseudoconvex domain $\Omega \subset \mathbb{C}^{n}, n \geq 2$, and $\zeta \in \partial \Omega$, find a neighbourhood $U_{\zeta}$ of $\zeta$ and a $\mathcal{C}^{2}$-smooth function $\rho_{\zeta} \in \operatorname{psh}\left(U_{\zeta}\right)$ such that

- $\rho_{\zeta}^{-1}\{0\}$ is a smooth hypersurface in $U_{\zeta}$ that is pseudoconvex from the side $U_{\zeta}^{-}:=\left\{z: \rho_{\zeta}(z)<0\right\} ;$ and

- $\rho_{\zeta}(\zeta)=0$, but $(\bar{\Omega} \backslash\{\zeta\}) \cap U_{\zeta} \varsubsetneqq U_{\zeta}^{-}$.

We shall call the triple $\left(\partial \Omega, U_{\zeta}, \rho_{\zeta}\right)$ a local bumping of $\Omega$ about $\zeta$. Fornaess and Sibony [10] devised a procedure of bumping to show that every boundary point of a finite-type domain in $\mathbb{C}^{2}$ admits a holomorphic peak function. The works [9, 12 ] are just some of the many applications of bumping in $\mathbb{C}^{2}$.

Going beyond $\mathbb{C}^{2}$, Diederich and Fornaess [7] have shown that if $\Omega$ is a bounded, pseudoconvex domain with real-analytic boundary, then local bumpings always exist about each $\zeta \in \partial \Omega$. However, the applications cited above rely on a second ingredient: that the bumpings constructed are, in some sense, well-adapted to the pair $(\Omega, \zeta)$. To be more specific, it is highly desirable for a local bumping $\left(\partial \Omega, U_{\zeta}, \rho_{\zeta}\right)$ to have the following two properties:

(B1) The orders of contact of $\partial \Omega \cap U_{\zeta}$ with $\rho_{\zeta}^{-1}\{0\}$ at $\zeta$ along various directions $V \in T_{\zeta}(\partial \Omega) \cap i T_{\zeta}(\partial \Omega)$ are the lowest possible.

2000 Mathematics Subject Classification. Primary 32F05, 32T25.

Key words and phrases. Bumping, finite-type domain, plurisubharmonic function, model domain, weighted-homogeneous function.

This work is supported in part by a grant from the UGC under DSA-SAP, Phase IV. 
(B2) The function $\rho_{\zeta}$ is as simple as possible and is explicitly known.

The difficulty with the results of [7] is that when $\Omega \Subset \mathbb{C}^{n}$ and $n \geq 3$, the order of contact between $\partial \Omega$ and $\rho_{\zeta}^{-1}\{0\}$ at $\zeta$ along certain complex-tangential directions can be very high. Furthermore, the great generality of the scope of [7] makes it very hard for an explicit equation for $\rho_{\zeta}$ to be discernable.

Yet, there has been some progress — which generalises the situation in [10] in achieving $(*)$ so that the local bumping has the properties (B1) and (B2). To appreciate this, we need to define the Catlin normal form for a pair $(\Omega, \zeta)$. To this end, we refer the reader to Catlin [3] for a definition of the Catlin multitype.

Definition 1.1. Let $\Omega$ be a pseudoconvex domain in $\mathbb{C}^{n+1}, n \geq 1$, having $\mathcal{C}^{\infty}$ smooth boundary and let $\zeta \in \partial \Omega$. Let $\left(1, m_{1}, \ldots, m_{n}\right)$ be the Catlin multitype of $\partial \Omega$ at $\zeta$ and suppose $m_{j}<\infty, j=1, \ldots, n$. Then, by [3, Main Theorem], there exists a local holomorphic coordinate system $\left(U_{\zeta} ; w, z_{1}, \ldots, z_{n}\right)$ centered at $\zeta$, which we shall call distinguished coordinates, such that

$$
\Omega \bigcap U_{\zeta}=\left\{(w, z) \in V_{\zeta}: \mathfrak{R e} w+P(z)+Q(z)+r(\mathfrak{I m} w, z)<0\right\},
$$

where $V_{\zeta}$ is a neighbourhood of $0 \in \mathbb{C}^{n+1}$, and where

- $P$ is an $\left(m_{1}, \ldots, m_{n}\right)$-homogeneous plurisubharmonic polynomial in $\mathbb{C}^{n}$ that has no pluriharmonic terms;

- $D^{\alpha} \bar{D}^{\beta} Q(0)=0$ whenever $(\alpha, \beta) \in \mathbb{N}^{n} \times \mathbb{N}^{n}$ such that $\sum_{j=1}^{n} m_{j}^{-1}\left(\alpha_{j}+\beta_{j}\right) \leq 1$; and

- There exists a smooth function $S$ defined about $0 \in \mathbb{R}$ such that

$$
\frac{r(x, z)-S(x)}{|x|}=o\left(\sqrt{\left|z_{1}\right|^{m_{1}}+\cdots+\left|z_{n}\right|^{m_{n}}}\right) \text { as }(x, z) \rightarrow 0 .
$$

Any presentation of $\Omega$ in a neighbourhood of $\zeta \in \partial \Omega$ having the form (1.1) will be called a Catlin normal form for $(\Omega, \zeta)$.

We remind the reader that, given an $n$-tuple $\Lambda=\left(\lambda_{1}, \ldots, \lambda_{n}\right), P$ is said to be $\Lambda$ homogeneous if $P\left(t^{1 / \lambda_{1}} z_{1}, \ldots, t^{1 / \lambda_{n}} z_{n}\right)=t P\left(z_{1}, \ldots, z_{n}\right) \forall z=\left(z_{1}, \ldots, z_{n}\right) \in \mathbb{C}^{n}$ and for every $t>0$. The behaviour of the the term $r$ above is already implicit in [3], but has been made explicit in [14 following an argument by Fornaess-Sibony [10].

Let the pair $(\Omega, \zeta)$ be as in Definition 1.1. If the model domain $\Omega_{P}:=\{(w, z) \in$ $\left.\mathbb{C} \times \mathbb{C}^{n}: \mathfrak{R e} w+P(z)<0\right\}$ - where $P$ is the polynomial occurring in (1.1) - is of finite type, then it was shown independently by $\mathrm{Yu}$ in [13], and by DiederichHerbort in [8], that one can make a holomorphic change of the $w$-coordinate, and find a neighbourhood $V_{\zeta}$ of $0 \in \mathbb{C}^{n+1}$ and an $\left(m_{1}, \ldots, m_{n}\right)$-homogeneous function $H \in \mathcal{C}^{1}\left(\mathbb{C}^{n}\right)$, that is positive away from 0 , such that $(P-H) \in \operatorname{psh}\left(\mathbb{C}^{n}\right)$, and

$$
(\bar{\Omega} \backslash\{\zeta\}) \bigcap U_{\zeta} \varsubsetneqq\left\{(w, z) \in V_{\zeta}: \mathfrak{R e} w+P(z)-H(z)<0\right\} .
$$

With $\Omega$ as above, whenever the model domain $\Omega_{P}$ associated to its Catlin normal form at some $\zeta \in \partial \Omega$ is of finite type, we say that $\Omega$ is $h$-extendible at $\zeta \in \partial \Omega$ (or, alternatively, that $(\Omega, \zeta)$ is h-extendible). It is evident that the bumping represented by the right-hand side of (1.2) also has the properties (B1) and (B2). Are such elementary model domains available when the pair $(\Omega, \zeta)$ is not $h$-extendible?

The answer to the above question is definitively, "No." Yu in [13] and DiederichHerbort in [8] have independently shown that: 
Fact 1.2. An $\left(m_{1}, \ldots, m_{n}\right)$-homogeneous $H$ as in (1.2) exists if and only if there are no complex subvarieties of $\mathbb{C}^{n}$ of positive dimension along which $P$ is harmonic.

The purpose of this paper is to show that if the domain $\Omega$ in question is in $\mathbb{C}^{3}$ and, for the chosen boundary point $\zeta$, the structure of the exceptional complex varieties along which $P$ - in the notation of (1.1) - is not too complicated, then one can construct a local bumping $\left(\partial \Omega, U_{\zeta}, \rho_{\zeta}\right)$ that has the properties (B1) and (B2) and, in particular, $\rho_{\zeta}$ is not much more complicated than the formula occurring in (1.2). The class of pointed domains $(\Omega, \zeta)$ we shall discuss is strictly larger than the class of $h$-extendible pairs. The precise definition will be given in the next section, but a representative of the class that we will study is:

\section{Example 1.3.}

$$
\Omega=\left\{(w, z) \in \mathbb{C} \times \mathbb{C}^{2}: \mathfrak{R e}(w)+\left|z_{1}\right|^{6}\left|z_{2}\right|^{2}+\left|z_{1}\right|^{8}+\frac{15}{7}\left|z_{1}\right|^{2} \mathfrak{R e}\left(z_{1}^{6}\right)+\left|z_{2}\right|^{10}<0\right\} .
$$

Note that $(\Omega, 0)$ is not an $h$-extendible pair because $P\left(z_{1}, z_{2}\right)=\left|z_{1}\right|^{6}\left|z_{2}\right|^{2}+\left|z_{1}\right|^{8}+$ $\frac{15}{7}\left|z_{1}\right|^{2} \mathfrak{R e}\left(z_{1}^{6}\right)$ (the above example is already in Catlin normal form at $\zeta=0$ ) is harmonic along the complex line $\left\{\left(z_{1}, z_{2}\right) \in \mathbb{C}^{2}: z_{1}=0\right\}$. The purpose of this paper is to construct model bumpings for domains of the class to which Example 1.3 belongs.

\section{Statement of Results}

We begin with some notation that is relevant to the definitions and results in this section. Let $P$ be an $\left(m_{1}, m_{2}\right)$-homogeneous plurisubharmonic polynomial on $\mathbb{C}^{2}$, $m_{1}, m_{2} \in \mathbb{Z}_{+}$, and define:

$$
\begin{aligned}
& \omega(P):=\left\{z \in \mathbb{C}^{2}: \mathfrak{H}_{\mathbb{C}}(P)(z) \text { is not strictly positive definite }\right\}, \\
& \mathfrak{C}(P):=\text { the set of all irreducible complex curves } X \subset \mathbb{C}^{2} \\
& \text { such that } P \text { is harmonic along the smooth part of } X \text {, } \\
& \mathcal{E}(P):=\text { the class of all curves of the form } \\
& \left\{\left(z_{1}, z_{2}\right): z_{1}^{m_{1} / \operatorname{gcd}\left(m_{1}, m_{2}\right)}=\xi z_{2}^{m_{2} / \operatorname{gcd}\left(m_{1}, m_{2}\right)}\right\}, \\
& \xi \in \widehat{\mathbb{C}} \text {, along which } P \text { is harmonic }
\end{aligned}
$$

(understanding that $\xi=\infty \Rightarrow P$ is harmonic along $\left\{\left(z_{1}, z_{2}\right) \in \mathbb{C}^{2}: z_{2}=0\right\}$ ), where $\mathfrak{H}_{\mathbb{C}}(P)(z)$ denotes the complex Hessian of $P$ at $z \in \mathbb{C}^{2}$. Here $P$ is the prototype for the lowest-weight polynomial that occurs in a Catlin normal form; see (1.1). The set $\mathcal{E}(P)$ will be the focus of our attention. This is because whenever $\mathfrak{C}(P) \neq \varnothing$, then $\mathcal{E}(P) \neq \varnothing$. This follows by making the obvious modifications - to allow for the fact that $P$ is $\left(m_{1}, m_{2}\right)$-homogeneous - to the proof of the following observation by Noell:

Result 2.1 (Lemma 4.2, [11]). Let $P$ be a homogeneous, plurisubharmonic, nonpluriharmonic polynomial in $\mathbb{C}^{n}, n \geq 2$. Suppose there exist complex-analytic varieties of positive dimension in $\mathbb{C}^{n}$ along which $P$ is harmonic. Then, there exist complex lines through the origin in $\mathbb{C}^{n}$ along which $P$ is harmonic.

Note that, in view of Fact [1.2, if a pair $(\Omega, \zeta)$ is not $h$-extendible, then $\mathcal{E}(P) \neq \varnothing$. Our claim is that, loosely speaking, the Levi-geometry around a boundary point $\zeta$ is tractable enough to enable simple bumpings about $\zeta$ for those non- $h$-extendible pairs $(\Omega, \zeta)$ for which a Catlin normal form has the property that $\mathfrak{C}(P)$ contains no 
complex curves other than those in $\mathcal{E}(P)$. However, we need to make this assertion precise. To do so, we need the following object. An $\left(m_{1}, m_{2}\right)$-wedge in $\mathbb{C}^{2}$ is defined to be a set $\mathcal{W}$ having the property that if $\left(z_{1}, z_{2}\right) \in \mathcal{W}$, then $\left(t^{1 / m_{1}} z_{1}, t^{1 / m_{2}} z_{2}\right) \in$ $\mathcal{W} \forall t>0$. The terms open $\left(m_{1}, m_{2}\right)$-wedge and closed $\left(m_{1}, m_{2}\right)$-wedge will have the usual meanings. Note that when $m_{1}=m_{2}=2 k$ (the true homogeneous case), an $\left(m_{1}, m_{2}\right)$-wedge is simply a cone. We also clarify that, in what follows, "finite type" will be used in the sense of D'Angelo. I.e., if $\partial \Omega$ is of finite type at $\zeta \in \partial \Omega$, we will mean that the 1-type $\Delta_{1}(\partial \Omega, \zeta)$ - see [5] for a definition - is finite. We can now present our key definition:

Definition 2.2. Let $\Omega$ be a pseudoconvex domain in $\mathbb{C}^{3}$ having $\mathcal{C}^{\infty}$-smooth boundary and let $\zeta \in \partial \Omega$. Let $\left(1, m_{1}, m_{2}\right)$ be the Catlin multitype of $\partial \Omega$ at $\zeta$. We say that $\Omega$ is almost $h$-extendible at $\zeta \in \partial \Omega$ (or, alternatively, that $(\Omega, \zeta$ ) is almost $h$-extendible) if $\partial \Omega$ is of finite type at $\zeta$ and, for some (consequently, for every) system of distinguished coordinates for $(\Omega, \zeta)$, the lowest-weight plurisubharmonic polynomial $P$ occurring in the normal form (1.1) has the following property:

$\omega(P) \backslash \bigcup_{X \in \mathcal{E}(P)} X$ contains no complex subvarieties of positive dimension and is well separated from $\bigcup_{X \in \mathcal{E}(P)} X$, i.e., there is a closed $\left(m_{1}, m_{2}\right)$-wedge

$\overline{\mathcal{W}}$ that contains $\omega(P) \backslash \bigcup_{X \in \mathcal{E}(P)} X$ and satisfies $\overline{\mathcal{W}} \cap\left(\bigcup_{X \in \mathcal{E}(P)} X\right)=\{0\}$.

Remark 2.3. It is evident from Definition 1.1 that distinguished coordinates $\left(U_{\zeta} ; w, z_{1}, z_{2}\right)$ need not be unique. However, it is easy to see that for each $P$ (i.e., relative to each system of distinguished coordinates), whether $\mathcal{E}(P)=\varnothing$ or $\mathcal{E}(P) \neq \varnothing$, and whether or not $\omega(P) \backslash \bigcup_{X \in \mathcal{E}(P)} X$ is well separated from $\bigcup_{X \in \mathcal{E}(P)} X$ in the latter case, is independent of the choice of distinguished coordinates. Furthermore, $\left(m_{1}, m_{2}\right) \in \mathbb{Z}_{+} \times \mathbb{Z}_{+}$. This is not evident from the definition of the Catlin multitype, but is a consequence of [3, Theorem 2.2].

Remark 2.4. Observe that if $(\Omega, \zeta)$ is $h$-extendible (in which case $\mathcal{E}(P)=\varnothing$ ), then it is almost $h$-extendible.

We must record one notational point: given a system of distinguished coordinates $\left(U_{\zeta} ; w, z_{1}, \ldots, z_{n}\right), w$ and $z_{j}, j=1, \ldots, n$, will interchangably denote the standard coordinates of $\mathbb{C} \times \mathbb{C}^{n}$ in which the normal form is presented as well as the components of an injective holomorphic map $\left(w, z_{1}, \ldots, z_{n}\right):\left(U_{\zeta}, \zeta\right) \longrightarrow\left(\mathbb{C}^{n+1}, 0\right)$. The pairs $(\Omega, \zeta)$ for which we can state our main theorem will be required to satisfy the following mild condition:

$(* *)$ For each $X \in \mathcal{E}(P)$ and each $x \in(X \backslash\{(0,0)\}) \bigcap \operatorname{Dom}(Q)$

$$
\operatorname{Ord}\left(\left.P\right|_{\mathcal{N}^{x}}-P(x), x\right) \leq \operatorname{Ord}\left(\left.Q\right|_{\mathcal{N}^{x}}-Q(x), x\right),
$$

and, if $R>0$ is so small that $\overline{\mathbb{B}^{3}(0 ; R)} \subset\left(w, z_{1}, z_{2}\right)\left(U_{\zeta}\right)$, then

$$
|r(\mathfrak{I m} w, z)| \lesssim|\mathfrak{I m} w|^{\Delta_{1}(\partial \Omega)+\frac{1}{\operatorname{lcm}\left(m_{1}, m_{2}\right)}} \quad \forall(w, z) \in \mathbb{B}^{3}(0 ; R),
$$

where $\left(m_{1}, m_{2}\right)$ and $(P, Q, r)$ are determined by the distinguished coordinates $\left(U_{\zeta} ; w, z_{1}, z_{2}\right)$, and $\mathcal{N}^{x}$ denotes the complex line in $\mathbb{C}_{z}^{2}$ that is normal to the curve $X$ at $x$. We have abbreviated the 1-type $\Delta_{1}(\partial \Omega, \zeta)$ to $\Delta_{1}(\partial \Omega)$.

The condition $(* *)$ might seem like a severe restriction on the class of almost $h$ extendible pairs, so a couple of explanations are in order. Given a point $a \in \mathbb{C}$ and 
a smooth function $F$ defined in a neighbourhood of $a$ such that $F(a)=0$, we write:

$\operatorname{Ord}(F, a):=\min \left\{n \in \mathbb{Z}_{+}:\right.$some monomial of degree $n$ in the Taylor expansion of $F$ around $a$ has non-zero coefficient $\}$.

Now, concerning the first part of $(* *):\left(\left.P\right|_{\mathcal{N}^{x}}-P(x)\right)$ may well vanish to infinite order in some real direction in $\mathcal{N}^{x}$, but we do not require $\left(\left.Q\right|_{\mathcal{N}^{x}}-Q(x)\right)$ to have the same decay along such a direction if $\operatorname{Ord}\left(\left.P\right|_{\mathcal{N}^{x}}-P(x)\right)<\infty$. (Refer also to the short remark just after Theorem [2.5.) As for $(* *)$ : we do not require the condition on $r$ in order to obtain a result in spirit of Theorem 2.5. But there is a pragmatic reason for imposing this condition; the reader is referred to Remark 2.7 following Theorem 2.5.

We can now state the main theorem of this paper.

Theorem 2.5. Let $\Omega$ be a pseudoconvex domain in $\mathbb{C}^{3}$ having $\mathcal{C}^{\infty}$-smooth boundary and let $\zeta \in \partial \Omega$. Assume that $\Omega$ is almost $h$-extendible at $\zeta$. Let $\left(1, m_{1}, m_{2}\right)$ be the Catlin multitype of $\partial \Omega$ at $\zeta$. Let

$$
\Omega \bigcap U_{\zeta}=\left\{(w, z) \in V_{\zeta}: \mathfrak{R e} w+P(z)+Q(z)+r(\mathfrak{I m} w, z)<0\right\}
$$

be the local representation of $\Omega$ with respect to a system of distinguished coordinates $\left(U_{\zeta} ; w, z_{1}, z_{2}\right)$ (here, and below, $\left.z:=\left(z_{1}, z_{2}\right)\right)$. If $\mathcal{E}(P) \neq \varnothing$, then:

1) $\mathcal{E}(P)$ is a finite set, denoted as $\left\{X_{1}, \ldots, X_{n}\right\}$.

2) Suppose $(\Omega, \zeta)$ satisfies $(* *)$. Then, we can find a system of holomorphic coordinates $\left(U_{\zeta} ; W, Z_{1}, Z_{2}\right)$ in such a way that $Z=z$, and construct a plurisubharmonic function $G \in \mathcal{C}^{\infty}\left(\mathbb{C}^{2} \backslash\{0\}\right) \cap \mathcal{C}^{2}\left(\mathbb{C}^{2}\right)$ whose orders of vanishing at $0 \in \mathbb{C}^{2}$ along various complex directions in $\mathbb{C}^{2}$ are explicitly known and such that

$$
(\bar{\Omega} \backslash\{0\}) \bigcap \mathbb{B}^{3}(0 ; R) \varsubsetneqq\left\{(W, z) \in \mathbb{C}^{3}: \mathfrak{R e} W+G(z)<0\right\} \bigcap \mathbb{B}^{3}(0 ; R)
$$

for some $R>0$.

The function $G$ has the following description. There exist a non-negative function $\mathcal{H}_{0} \in \mathcal{C}^{\infty}\left(\mathbb{C}^{2} \backslash\{0\}\right) \cap \mathcal{C}^{2}\left(\mathbb{C}^{2}\right)$ that is $\left(m_{1}, m_{2}\right)$-homogeneous; subharmonic functions $v_{j} \in \mathcal{C}^{\infty}(\mathbb{C} \backslash\{0\}) \cap \mathcal{C}^{2}(\mathbb{C})$ that are homogeneous of degree $2 d_{j}, j=1, \ldots, N$, and are strictly subharmonic away from $0 \in \mathbb{C}$; and closed $\left(m_{1}, m_{2}\right)$-wedges $\overline{\mathfrak{W}}_{j}^{1}$ and $\overline{\mathcal{W}}_{j}^{2}$ satisfying

such that:

$$
\overline{\mathcal{W}}_{j}^{1} \backslash\{0\} \varsubsetneqq \operatorname{int}\left(\overline{\mathcal{W}}_{j}^{2}\right), \quad j=1, \ldots, N,
$$

- $\mathcal{H}_{0}^{-1}\{0\}=\bigcup_{j=1}^{N} X_{j}$.

- For each $j \leq N, G(z)=\left(P-\mathcal{H}_{0}\right)(z)+v_{j}\left(z_{k(j)}\right) \forall\left(z_{1}, z_{2}\right) \in \overline{\mathcal{W}}_{j}^{1} \cap \mathbb{B}^{2}(0 ; R)$, where $k(j)=1$ or 2 depending on $j=1, \ldots, N$.

- $G\left(z_{1}, z_{2}\right)=\left(P-\mathcal{H}_{0}\right)\left(z_{1}, z_{2}\right) \forall\left(z_{1}, z_{2}\right) \in\left(\mathbb{C}^{2} \backslash \cup_{j=1}^{N} \overline{\mathcal{W}}_{j}^{2}\right) \cap \mathbb{B}^{2}(0 ; R)$.

Remark 2.6. We have given a part of the explanation for the assertion that the first part of condition $(* *)$ is mild. Another reason for this assertion is that that condition can be dispensed with. I.e., even without that condition, a bumping theorem similar to Theorem 2.5 can be deduced. However, the model that one would get would have a more complicated defining function than the one above. Since the main point of Theorem 2.5 is to construct the simplest bumpings in the almost $h$-extendible case, we will assume that the the first part of $(* *)$ is in effect. 
Remark 2.7. The second part of the condition $(* *)$ can also be dispensed with. The theorem that one obtains without this assumption differs from Theorem 2.5 in the following respect: in addition to all the objects asserted to exist in Theorem 2.5, there exist polynomials $q_{j} \in \mathbb{C}\left[z_{1}, z_{2}\right], j=1, \ldots, N$, with $\operatorname{deg}\left(q_{j}\right)<2 d_{j}$ such that

$$
G(z)=\left(P-\mathcal{H}_{0}\right)(z)+\mathfrak{R e} q_{j}(z)+v_{j}\left(z_{k(j)}\right) \forall\left(z_{1}, z_{2}\right) \in \overline{\mathcal{W}}_{j}^{1} \bigcap \mathbb{B}^{2}(0 ; R),
$$

where $k(j)=1$ or 2 depending on $j=1, \ldots, N$. This latter version takes some extra effort to prove but has the advantage that, for domains in $\mathbb{C}^{3}$, it subsumes the bumping results in [8] and [13]. However, it is not clear whether the right-hand side of the above equation is simple enough to be useful in applications. In contrast, if we study unbounded domains of the form (1.1) with $Q \in \mathcal{C}^{\infty}\left(\mathbb{C}^{2}\right)$ and $r \equiv 0$, then Theorem 2.5 can be applied to several problems on such domains. For example, using Theorem 2.5] one can extend (in $\mathbb{C}^{3}$ ) [4, Theorem 1.2] by Chen-KamimotoOhsawa to a much wider class of unbounded domains (which we shall address in a forthcoming work). To summarise: because of the utility of the bumpings we get from Theorem 2.5, we prefer to restrict attention to domains that satisfy the second part of $(* *)$.

We ought to mention that the idea of "almost $h$-extendibility" comes from [2]. The property that the polynomial $P$ occurring in Definition 2.2 possesses is termed Property (A) in [2]. In fact, some of the preliminary steps in our proof of Theorem 2.5 are variations on the work in 2. Before we conclude this section, let us glance at the key ideas involved in the proof of Theorem 2.5. The four primary ingredients in our proof are as follows:

- Step 1: As in 2], we begin by studying the true homogeneous case, i.e. when the Catlin multitype of $\partial \Omega$ at $\zeta$ is $(1,2 k, 2 k), k \geq 2$. For each complex line $X \in \mathcal{E}(P)$, we can find a closed cone $\overline{\mathcal{K}}^{X}$ and a function $H_{X}$ that is supported in $\overline{\mathcal{K}}^{X}$ such that $\left(P-\delta H_{X}\right)$ is a bumping of $P$, for $\delta>0$ sufficiently small, inside the aforementioned cone. The departure from [2] here is that $H_{X}$ must be constructed with greater delicacy so that the precise orders of decay of $H_{X}$, as one approaches its zero set, are known. This involves some new ideas.

- Step 2: We know from [2, Proposition 1] that, in the homogeneous case, there are finitely many complex lines $X_{1}, \ldots, X_{N} \in \mathcal{E}(P)$. We use pseudoconvexity of $\Omega$, plus the hypothesis of $\partial \Omega$ being of finite type at $\zeta$, to extract summands from the Taylor expansion of $(P+Q)$ (see Definition 1.1) that constitute homogeneous subharmonic polynomials corresponding to each $X_{j}, j=1, \ldots, N$. A well-known prescription is used to bump these polynomials, which yields the functions $v_{1}, \ldots, v_{N}$ of Theorem 2.5.

- Step 3: The properties of $P$ allow us to patch together all the functions constructed up to this point in an appropriate manner. This yields the function $G$ of Theorem 2.5. We then account for those terms in the Taylor expansion of $(P+Q)$ that are not already in $\left.G\right|_{\mathcal{K}^{X}}$ so that we can eventually conclude that $G(z)<(P+Q)(z) \forall z \in \mathbb{B}^{2}(0 ; R) \backslash\{(0,0)\}$ for some $R>0$ sufficiently small.

- Step 4: The analogous results for the case when the Catlin multitype of $\partial \Omega$ at $\zeta$ is $\left(1, m_{1}, m_{2}\right), m_{1} \neq m_{2}$, are obtained by applying an appropriate proper holomorphic map that pulls back the Catlin normal form of $(\Omega, \zeta)$ to a 
domain to which the above ideas can be applied. A final change of coordinate accounts for the terms in the Taylor expansion of $r$ (see Definition 1.1).

The constructions described in the first step of the above procedure will be explained in Section 4. The second and the third steps represent the key technical proposition of this paper. They constitute Proposition 5.3, which will be presented in Section 5 below. The final part of the above analysis will be found in Section 6

\section{TECHNICAL PRELIMINARIES}

The purpose of this section is to list some results that we shall apply many times, in the sections that follow, to accomplish specific technical tasks.

We begin by stating a proposition that is essential for the proof of Theorem 2.5, and from which Part (1) of Theorem 2.5 follows almost immediately.

Result 3.1 (Proposition 1, 2]). Let $P$ be a plurisubharmonic, non-pluriharmonic polynomial in $\mathbb{C}^{2}$ that is homogeneous of degree $2 k$. Then, there are at most finitely many complex lines passing through $0 \in \mathbb{C}^{2}$ along which $P$ is harmonic.

The next result is a mild refinement of [10, Lemma 2.4] by Fornaess \& Sibony. In fact, we do not need to alter anything in the proof of [10, Lemma 2.4]. The refinement lies in stating explicitly, in Part (c) below, a property of the FornaessSibony construction of which there was no need in [10], but which we will need in Section 4 .

Result 3.2. Let $U: \mathbb{C} \rightarrow \mathbb{R}$ be a real-analytic, subharmonic, non-harmonic function that is homogeneous of degree $j$. Assume that

$$
\left\{\theta_{1}, \ldots, \theta_{M}\right\}:=\left\{\theta \in[0,2 \pi): \triangle U\left(e^{i \theta}\right)=0\right\} \subset(0,2 \pi) .
$$

Let $\sigma_{0}>0$ be so small that $\left[\theta_{k}-\sigma_{0}, \theta_{k}+\sigma_{0}\right] \subset(0,2 \pi)$ and $\left[\theta_{k}-\sigma_{0}, \theta_{k}+\sigma_{0}\right] \bigcap\left[\theta_{k+1}-\right.$ $\left.\sigma_{0}, \theta_{k+1}+\sigma_{0}\right]=\varnothing, k=1, \ldots M-1$. Next, define:

$$
\mathcal{S}_{k}(\sigma):=\left\{r e^{i \theta}: r \geq 0, \theta \in\left[\theta_{k}-\sigma, \theta_{k}+\sigma\right]\right\}, \quad \sigma \in\left(0, \sigma_{0}\right) .
$$

There exist positive constants

- $C_{1} \equiv C_{1}(U)$, which varies polynomially in $\sup _{|z|=1}|U(z)|$;

- $C_{2} \equiv C_{2}(U, \sigma)$, which varies polynomially in $\sup _{|z|=1}|U(z)|$ and $\sigma \in\left(0, \sigma_{0}\right)$; and a $2 \pi$-periodic function $h \in \mathcal{C}^{\infty}(\mathbb{R})$ such that:

a) $0<h(x) \leq 1 \forall x \in \mathbb{R}$.

b) $\triangle\left(U-\delta|\cdot|{ }^{j} h \circ \operatorname{Arg}(\cdot)\right)(z) \geq \delta C_{1}|z|^{j-2} \forall z \in \mathbb{C}$ and $\forall \delta: 0<\delta \leq 1$.

c) $\triangle\left(U-\delta|\cdot|{ }^{j} h \circ \operatorname{Arg}(\cdot)\right)(z) \geq C_{2}|z|^{j-2} \forall z \in \mathbb{C} \backslash\left(\bigcup_{k=1}^{M} \mathcal{S}_{k}(\sigma)\right)$ and $\forall \sigma \in$ $\left(0, \sigma_{0}\right)$, independent of $\delta$.

(Here $\operatorname{Arg}(\cdot)$ refers to any continuous branch of the argument.)

Remark 3.3. The $\delta>0$ appearing in the above lemma must not be confused with the $\delta$ appearing in the statement [10, Lemma 2.4]. The latter $\delta$ is a universal constant which is a component of the constant $C_{1}(U)$ in our notation. If we denote the $\delta$ of [10, Lemma 2.4] by $\delta_{\text {univ }}$, then our $C_{1}(U)$ is a polynomial function of $\delta_{\text {univ }}$ and

$$
\text { (in the notation of [10]) }\|U\|:=\sup _{|z|=1}|U(z)| \text {. }
$$

The last technical item is a Levi-form calculation. We will need this result in Section 4. 
Lemma 3.4. Let $P\left(z_{1}, z_{2}\right)$ be a plurisubharmonic, non-pluriharmonic polynomial that is homogeneous of degree $2 k, k \geq 2$, and assume that $P(0, \cdot) \equiv 0$. Write

$$
P\left(z_{1}, z_{2}\right)=\sum_{j=\mu}^{2 k} Q_{j}\left(z_{1}, z_{2}\right),
$$

where each $Q_{j}$ is the sum of all monomials of $P$ that involve powers of $z_{1}$ and $\bar{z}_{1}$ having total degree $j, \mu \leq j \leq 2 k$. Then $Q_{\mu}$ is plurisubharmonic.

Proof. We start with a Levi-form calculation. For $\zeta, w \in \mathbb{C}$, let us write

$$
\begin{aligned}
w & =|w| e^{i \phi}, \\
\zeta & =|\zeta| e^{i \alpha}, \text { where } \phi, \alpha \in \mathbb{T}:=\mathbb{R} / 2 \pi \mathbb{Z} .
\end{aligned}
$$

With this notation, the Levi-form of $P$ at the points $(\zeta w, w)$ can be written as

$$
\begin{array}{r}
\mathfrak{L} P(\zeta w, w ; v)=|w|^{2(k-1)}|\zeta|^{\mu-2} \times\left(\begin{array}{ll}
v_{1} & \zeta v_{2}
\end{array}\right)\left(\begin{array}{cc}
T_{11}(\phi, \alpha) & T_{12}(\phi, \alpha) \\
\overline{T_{12}(\phi, \alpha)} & T_{22}(\phi, \alpha)
\end{array}\right)\left(\begin{array}{c}
\overline{v_{1}} \\
\overline{\zeta v_{2}}
\end{array}\right) \\
+|w|^{2(k-1)} O\left(|\zeta|^{\mu-1}\left|v_{1}\right|^{2},|\zeta|^{\mu}\left|v_{1} v_{2}\right|,|\zeta|^{\mu+1}\left|v_{2}\right|^{2}\right) \geq 0,
\end{array}
$$

where $T_{11}, T_{12}$ and $T_{22}$ are trigonometric polynomials obtained when $\mathfrak{L} Q_{\mu}(\zeta w, w ; v)$ is written out relative to the polar coordinates defined above. We first note that as $P \in \operatorname{psh}\left(\mathbb{C}^{2}\right)$, whence $\mu \geq 2, \mathfrak{L} Q_{\mu}((0, \cdot) ; \cdot) \geq 0$. Let us assume that $Q_{\mu} \notin \operatorname{psh}\left(\mathbb{C}^{2}\right)$. Then, there exist $\zeta_{0} \neq 0, w_{0} \neq 0$ and a vector $V=\left(V_{1}, V_{2}\right) \in \mathbb{C}^{2}$ such that

$$
\mathfrak{L} Q_{\mu}\left(\zeta_{0} w_{0}, w_{0} ; V\right)=-C<0 .
$$

Now, for each $r>0$, define $W_{r}:=\left(r V_{1}, V_{2}\right)$. From the expression for the Levi-form of $Q_{\mu}$ in (3.1) above, it clear that

$$
\mathfrak{L} Q_{\mu}\left(r \zeta_{0} w_{0}, w_{0} ; W_{r}\right)=r^{\mu} \mathfrak{L} Q_{\mu}\left(\zeta_{0} w_{0}, w_{0} ; V\right)=-C r^{\mu} .
$$

Notice that by the definition of $Q_{l}$,

$$
\partial_{j \bar{k}}^{2} Q_{l}\left(r \zeta_{0} w_{0}, w_{0}\right) W_{r, j} \overline{W_{r, k}}=O\left(r^{l}\right) \quad \forall l \geq \mu+1, j, k=1,2 .
$$

Combining this fact with (3.2), we see that there exists a positive constant $\delta \ll 1$ such that

$$
\mathfrak{L} P\left(r \zeta_{0} w_{0}, w_{0} ; W_{r}\right)=-C r^{\mu}+O\left(r^{\mu+1}\right)<0 \quad \forall r \in(0, \delta) .
$$

But this contradicts the plurisubharmonicity of $P$. Hence our earlier assumption must be false, and $Q_{\mu}$ is plurisubharmonic.

\section{Homogeneous polynomials: bumping around $\mathcal{E}(P)$}

This section is devoted to making precise the ideas presented in Step 1 of the outline given in Section 2. In order to state the main result of this section cleanly, we need the following notation: for any $\xi \in \mathbb{C}, \mathcal{K}(\xi ; \varepsilon)$ will denote the open cone

$$
\mathcal{K}(\xi ; \varepsilon):=\left\{\left(z_{1}, z_{2}\right) \in \mathbb{C}^{2}:\left|z_{1}-\xi z_{2}\right|<\varepsilon\left|z_{2}\right|\right\} .
$$

Note that $\mathcal{K}(\xi ; \varepsilon)$ is a conical neighbourhood of the punctured complex line $\left\{\left(z_{1}=\right.\right.$ $\left.\left.\xi z_{2}, z_{2}\right): z_{2} \in \mathbb{C} \backslash\{0\}\right\}$. As stated in Section 2, the initial ideas in the proof of the proposition below are similar to those in the proof of [2, Proposition 2]. The departure from [2] here is represented by the estimate in Part $(a)$ of Proposition 4.2 below. This estimate is a more precise statement than Part (a) of [2, Proposition 2]. 
Establishing this requires a more delicate construction, which relies, in part, on the following result:

Result 4.1 (Theorem 3, 2]). Let $Q\left(z_{1}, z_{2}\right)$ be a plurisubharmonic, non-harmonic polynomial that is homogeneous of degree $2 p$ in $z_{1}$ and $2 q$ in $z_{2}$. Then, $Q$ is of the form

$$
Q\left(z_{1}, z_{2}\right)=U\left(z_{1}^{d} z_{2}^{D}\right),
$$

where $d, D \in \mathbb{Z}_{+}$and $U$ is a homogeneous, subharmonic, non-harmonic polynomial.

A comment about the hypothesis imposed on $P$ in the result below: the $P$ below is the prototype for the polynomial $P$ described in Definition 1.1 under the following assumptions:

- The pair $(\Omega, \zeta)$ is such that the Catlin multitype of $\partial \Omega$ at $\zeta$ is $(2 k, 2 k)$.

- $\Omega$ is almost $h$-extendible at $\zeta$ with $\mathcal{E}(P) \neq \varnothing$.

These are encoded in the hypothesis about $\left.P\right|_{\mathcal{K}(0 ; \varepsilon) \backslash L}$. In this prototype, $L$ must be viewed as a complex line belonging to $\mathcal{E}(P)$.

Proposition 4.2. Let $P\left(z_{1}, z_{2}\right)$ be a plurisubharmonic polynomial that is homogeneous of degree $2 k, k \geq 2$, contains no pluriharmonic terms, and such that

$$
P\left(z_{1}, z_{2}\right)=\sum_{j=\mu}^{2 k} Q_{j}\left(z_{1}, z_{2}\right), \quad \mu>0,
$$

where each $Q_{j}$ is the sum of all monomials of $P$ that involve powers of $z_{1}$ and $\bar{z}_{1}$ having total degree $j$. Write $L:=\left\{\left(z_{1}, z_{2}\right): z_{1}=0\right\}$, and assume that there exists an $\varepsilon>0$ such that $P$ is strictly plurisubharmonic in the cone $(\mathcal{K}(0 ; \varepsilon) \backslash L)$. Then, there exist constants $c, \sigma>0$ - which depend only on $P$ - and a non-negative function $H \in \mathcal{C}^{\infty}\left(\mathbb{C}^{2} \backslash\{0\}\right) \cap \mathcal{C}^{2}\left(\mathbb{C}^{2}\right)$ that is homogeneous of degree $2 k$ such that:

a) $H\left(z_{1}, z_{2}\right)>c\left|z_{1}\right|^{\mu}\left|z_{2}\right|^{2 k-\mu}$ when $0<\left|z_{1}\right|<\sigma\left|z_{2}\right|$.

b) For every $\delta: 0<\delta \leq 1,(P-\delta H)$ is strictly plurisubharmonic on $(\mathcal{K}(0 ; \sigma) \backslash L)$. Furthermore, there is a constant $B \equiv B(t)>0$ that is independent of $\delta \in(0,1]$ - and depends only on $\sigma$ and $t$ - such that, whenever $t \in(0,1)$,

$$
\begin{aligned}
\mathfrak{L}(P-\delta H)\left(z ;\left(V_{1}, V_{2}\right)\right) \geq & B(t)\|z\|^{2(k-1)}\|V\|^{2} \\
& \forall z: t \sigma\left|z_{2}\right|<\left|z_{1}\right|<\sigma\left|z_{2}\right|, \quad \forall V \in \mathbb{C}^{2},
\end{aligned}
$$

for every $\delta: 0<\delta \leq 1$.

Proof. From Result 4.1, we deduce that there exist a homogeneous, subharmonic, non-harmonic polynomial $U$; and $a, b \in \mathbb{Z}_{+}$such that $Q_{\mu}\left(z_{1}, z_{2}\right)=U\left(z_{1}^{a} z_{2}^{b}\right)$. Then, adopting the notation used in the proof of Lemma 3.4 above, we see that the Leviform of $P$ at the points $(\zeta w, w)$ has the form:

$$
\begin{aligned}
& \mathfrak{L} P(\zeta w, w ; V)=U_{\xi \bar{\xi}}\left(\zeta^{a} w^{a+b}\right) J(\zeta, w)\left|a V_{1}+b \zeta V_{2}\right|^{2} \\
& +|w|^{2(k-1)} \sum_{j=\mu+1}^{2 k}|\zeta|^{j-2} \times\left(\begin{array}{ll}
V_{1} & \left.\zeta V_{2}\right)
\end{array}\left(\begin{array}{ll}
T_{11}^{j}(\phi, \alpha) & T_{12}^{j}(\phi, \alpha) \\
\overline{T_{12}^{j}(\phi, \alpha)} & T_{22}^{j}(\phi, \alpha)
\end{array}\right)\left(\begin{array}{c}
\overline{V_{1}} \\
\overline{\zeta V_{2}}
\end{array}\right)\right. \\
& \equiv U_{\xi \bar{\xi}}\left(\zeta^{a} w^{a+b}\right) J(\zeta, w)\left|a V_{1}+b \zeta V_{2}\right|^{2}+|w|^{2(k-1)} \mathcal{R}(\zeta, \phi ; V),
\end{aligned}
$$

where $J(\zeta, w):=|\zeta|^{2(a-1)}|w|^{2(a+b-1)} ; T_{11}^{j}, T_{12}^{j}$ and $T_{22}^{j}$ are trigonometric polynomials obtained when $\mathfrak{L} Q_{j}(\zeta w, w ; V)$ is written out in polar coordinates as described in the 
proof of Lemma 3.4, and where $\mathcal{R}: \mathbb{C} \times \mathbb{T} \times \mathbb{C}^{2} \longrightarrow \mathbb{R}$. Define the matrix-valued function $\mathcal{M}: \mathbb{C} \times \mathbb{T} \longrightarrow \mathbb{C}^{2 \times 2}$ by the relation

$$
\mathcal{R}(\zeta, \phi ; V)=\left\langle\mathcal{M}(\zeta, \phi)\left(V_{1}, \zeta V_{2}\right),\left(V_{1}, \zeta V_{2}\right)\right\rangle,
$$

where the inner product above is the standard Hermitian inner product on $\mathbb{C}^{2}$. We wish to construct the $H$ mentioned in the statement of this proposition by applying Result 3.2 to the polynomial $U$. Consequently, establishing Part $(b)$ of this proposition would require some estimates on the quantity $\mathcal{R}$. We shall begin with this task and divide our proof into three steps.

Step 1. Positivity estimates on $\mathcal{R}$ when $V$ lies in a conical neighbourhood of $\left\{V \in \mathbb{C}^{2}: a V_{1}+b \zeta V_{2}=0\right\}$

Consider the set

$$
\mathcal{S}_{1}:=\left\{(\zeta, \phi) \in \mathbb{C} \times \mathbb{T}: \operatorname{Ker}(\mathcal{M}(\zeta, \phi)) \supseteq\left\{\left(V_{1}, \zeta V_{2}\right) \in \mathbb{C}^{2}: a V_{1}+b \zeta V_{2}=0\right\}\right\},
$$

which is clearly a real-analytic subvariety of $\mathbb{C} \times \mathbb{T}$. Assume that $\mathcal{S}_{1} \bigcap\left(D(0 ; r)^{*} \times \mathbb{T}\right) \neq$ $\varnothing \forall r>0$. Here, and later in this proof, $D(0 ; r)^{*}$ shall denote the punctured disc $D(0 ; r) \backslash\{0\} \subset \mathbb{C}$. Then, there exists a sequence $\left\{\left(\zeta_{n}, \phi_{n}\right)\right\}_{n \in \mathbb{N}} \subset \mathbb{C} \times \mathbb{T}$ such that $\zeta_{n} \longrightarrow 0$ as $n \rightarrow+\infty$ and

$$
\mathcal{M}\left(\zeta_{n}, \phi_{n}\right)\left(\begin{array}{c}
-b \\
a
\end{array}\right)=0 \forall n \in \mathbb{N} .
$$

This implies, in view of (4.1), that

$$
\mathfrak{L} P\left(\zeta_{n} e^{i \phi_{n}}, e^{i \phi_{n}} ;\left(-b \zeta_{n}, a\right)\right)=0 \quad \forall n \in \mathbb{N} .
$$

But this means that $P$ is not strictly plurisubharmonic on the set of points $\left\{\left(\zeta_{n} e^{i \phi_{n}}, e^{i \phi_{n}}\right): n \in \mathbb{N}\right\}$, which has non-empty intersection with $(\mathcal{K}(0 ; \varepsilon) \backslash L)$. This contradicts our hypothesis, whence our assumption on $\mathcal{S}_{1}$ is false. Therefore, $\exists r_{1} \in(0, \varepsilon)$ such that $\mathcal{S}_{1}^{r_{1}}:=\mathcal{S}_{1} \bigcap\left(D\left(0 ; r_{1}\right)^{*} \times \mathbb{T}\right)=\varnothing$

Let us define the following auxiliary objects:

$$
\begin{aligned}
\widetilde{\mathcal{R}}(\zeta, \phi ; V) & :=|\zeta|^{2}\langle\mathcal{M}(\zeta, \phi) V, V\rangle, \\
\mathfrak{u} & :=(-b, a) /\|(-b, a)\| .
\end{aligned}
$$

From the following facts:

- $P$ is strictly plurisubharmonic on $(\mathcal{K}(0 ; \varepsilon) \backslash L)$;

- $\mathcal{S}_{1}^{r_{1}}=\varnothing$, with $r_{1}<\varepsilon$;

- $\mathfrak{L} Q_{\mu}((\zeta \cdot, \cdot) ;(-b \zeta, a)) \equiv 0$;

we conclude that $\mathcal{R}(\zeta, \phi ;(-b \zeta, a))>0$ on $D\left(0 ; r_{1}\right)^{*} \times \mathbb{T}$. Owing to the real-analyticity of $\mathcal{R}$, we can find an exponent $\eta: \mu+1 \leq \eta \leq 2 k$, and a constant $\sigma_{1}>0$ such that:

(i) $\mathcal{R}(\zeta, \phi ;(-b \zeta, a)) \geq \sigma_{1}|\zeta|^{\eta} \forall(\zeta, \phi) \in D\left(0 ; 3 r_{1} / 4\right) \times \mathbb{T}$.

(ii) For each $\phi \in \mathbb{T}$

$$
\liminf _{\zeta \rightarrow 0} \frac{\mathcal{R}(\zeta, \phi ;(-b \zeta, a))}{|\zeta|^{\eta}}>\sigma_{1} / 2
$$

In view of the identity

$$
\frac{\mathcal{R}(\zeta, \phi ;(-b \zeta, a))}{a^{2}+b^{2}}=\widetilde{\mathcal{R}}(\zeta, \phi ; \mathfrak{u})
$$


the bounds in $(i)$ and $(i i)$ above tell us that there is a $D\left(0 ; 3 r_{1} / 4\right) \times \mathbb{T} \times S^{3}$-open neighbourhood, say $\mathcal{U}$, of $D\left(0 ; 3 r_{1} / 4\right) \times \mathbb{T} \times\left\{\mathfrak{u} e^{i t}: t \in \mathbb{R}\right\}$ such that

$$
\widetilde{\mathcal{R}}(\zeta, \phi ; V) \geq \frac{\sigma_{1}}{4\left(a^{2}+b^{2}\right)}|\zeta|^{\eta} \forall(\zeta, \phi, V) \in \mathcal{U}
$$

Here, and later in this proof, $S^{3}$ shall denote the unit (Euclidean) sphere in $\mathbb{C}^{2}$. From the last inequality, it follows that there exists a small $S^{3}$-open neighbourhood $\mathcal{W}$ of $\left\{\mathfrak{u} e^{i t}: t \in \mathbb{R}\right\}$ such that

$$
\widetilde{\mathcal{R}}(\zeta, \phi ; V) \geq \frac{\sigma_{1}}{4\left(a^{2}+b^{2}\right)}|\zeta|^{\eta} \forall(\zeta, \phi, V) \in \overline{D\left(0 ; r_{1} / 2\right)} \times \mathbb{T} \times \mathcal{W} .
$$

And finally, exploiting the relationship between $\widetilde{\mathcal{R}}$ and $\mathcal{R}$, we infer from (4.2) that there exists a small constant $\beta>0$ - which depends upon the cone determined by $\mathcal{W}$ - such that

$$
\begin{aligned}
\text { If }\left|a V_{1}+b \zeta V_{2}\right| & \leq \beta\left|a \zeta V_{2}-b V_{1}\right| \text { then } \\
\mathcal{R}(\zeta, \phi ; V) & \geq \frac{\sigma_{1}}{4\left(a^{2}+b^{2}\right)}|\zeta|^{\eta}\left(\left|\frac{V_{1}}{\zeta}\right|^{2}+\left|V_{2}\right|^{2}\right), \quad(\zeta, \phi) \in\left(\overline{D\left(0 ; r_{1} / 2\right)} \backslash\{0\}\right) \times \mathbb{T} .
\end{aligned}
$$

From this we can infer that, shrinking $\beta>0$ if necessary, if we define the closed cones

$$
\overline{\mathfrak{C}}_{\beta}(\zeta):=\left\{V \in \mathbb{C}^{2}:((b / a)-\beta)\left|\zeta V_{2}\right| \leq\left|V_{1}\right| \leq((b / a)+\beta)\left|\zeta V_{2}\right|\right\}
$$

(understanding $\beta$ to be smaller than $b / a$ ), then there are small constants $c_{1}, R_{1}>0$ such that

$$
\begin{aligned}
& \mathcal{R}(\zeta, \phi ; V) \geq c_{1}|\zeta|^{\eta-2}\left(\left|V_{1}\right|^{2}+\left|\zeta V_{2}\right|^{2}\right) \\
& \forall(\zeta, \phi, V) \in \underset{\widetilde{\zeta} \in \overline{D\left(0 ; R_{1}\right)}}{\bigcup}\{\widetilde{\zeta}\} \times \mathbb{T} \times \overline{\mathfrak{C}}_{\beta}(\widetilde{\zeta}) .
\end{aligned}
$$

Step 2. The structure of the set $\left\{(\zeta, w): w \zeta \neq 0\right.$ and $\left.U_{\xi \bar{\xi}}\left(\zeta^{a} w^{a+b}\right)=0\right\}$ and definition of $H$ when this set is non-empty

Let $\left\{\theta_{1}, \ldots, \theta_{M}\right\}$ be the set associated to $\triangle U=4 U_{\xi \bar{\xi}}$ as defined in Result [3.2. The arguments in this part of our proof are predicated on the assumption that $\left\{\theta_{1}, \ldots, \theta_{M}\right\} \neq \varnothing$. We will consider what to do when $\left\{\theta_{1}, \ldots, \theta_{M}\right\}=\varnothing$ in Step 3 . We compute:

$$
\begin{aligned}
\mathfrak{S}_{1} & :=\left\{(\zeta, w) \in \mathbb{C}^{2}: w \zeta \neq 0 \text { and } U_{\xi \bar{\xi}}\left(\zeta^{a} w^{a+b}\right)=0\right\} \\
& =\bigcup_{k=1}^{M} \bigcup_{r>0}\left\{(\zeta, w) \in \mathbb{C}^{2}: \zeta^{a} w^{a+b}=r e^{i \theta_{k}}\right\} .
\end{aligned}
$$

We can apply a simple computation to (4.4) to get:

$$
\mathfrak{S}_{1}=\bigcup_{\alpha \in \mathbb{R}} \bigcup_{k=1}^{M} \bigcup_{l=1}^{a+b}\left\{\left(r e^{i \alpha}, s e^{i \phi}\right): r, s>0, \phi=\frac{\theta_{k}-a \alpha+2 \pi l}{a+b}\right\} .
$$

In other words, for each fixed $\zeta \in \mathbb{C} \backslash\{0\}, \mathfrak{S}_{1} \bigcap(\{\zeta\} \times \mathbb{C})$ is a collection of radial segments. We now apply Result 3.2 to $U$ and let $h$ be the $2 \pi$-periodic function whose existence is asserted by Result 3.2. Let $m \in \mathbb{Z}_{+}$be such that $U$ is homogeneous of degree $2 m$. Define

$$
F\left(r e^{i \theta}\right):=r^{2 m} h(\theta) \forall r e^{i \theta} \in \mathbb{C} .
$$


Now set

$$
\begin{aligned}
& A_{k}:=\frac{\sup \left\{t>0: \triangle(U-\delta F)\left(e^{i \theta}\right)>\triangle U\left(e^{i \theta}\right) \forall \theta \in\left(\theta_{k}-t, \theta_{k}+t\right)\right\}}{2} \\
& k=1, \ldots M, \quad 0<\delta \leq 1 .
\end{aligned}
$$

The whole point of Part $(b)$ of Result 3.2 is that $A_{k}>0 \forall k \leq M$. It is obvious that $A_{k}$ is independent of $\delta$ because any lowering of $\triangle(U-\delta F)\left(e^{i \theta}\right)$ with respect to $\triangle U\left(e^{i \theta}\right)$ is determined only by the cut-off functions used to construct $h$.

Let us now define

$$
H\left(z_{1}, z_{2}\right):=F\left(z_{1}^{a} z_{2}^{b}\right) \forall\left(z_{1}, z_{2}\right) \in \mathbb{C}^{2} .
$$

Let us also set $A:=\min _{1 \leq k \leq M} A_{k}$. Refer to Result 3.2 for a definition of the sets $\mathcal{S}_{k}(A)$, which are just closed sectors in $\mathbb{C}$. If we now define the set $\omega \subset \mathbb{C}^{2}$ by

$$
\omega:=\bigcup_{\alpha \in \mathbb{R}} \bigcup_{k=1}^{M} \bigcup_{l=1}^{a+b}\left\{\left(r e^{i \alpha}, s e^{i \phi}\right): r, s \geq 0,\left|\phi-\frac{\theta_{k}-a \alpha+2 \pi l}{a+b}\right| \leq \frac{A}{a+b}\right\},
$$

then, comparing this with (4.5) and the definition of $A$, we infer:

$$
(\zeta, w) \in \omega \Longleftrightarrow \zeta^{a} w^{a+b} \in \mathcal{S}_{k}(A) \text { for some } k=1, \ldots, M .
$$

Then, from the definition of $A$, we conclude that

$$
\begin{aligned}
\mathfrak{L}\left(Q_{\mu}-\delta H\right)(\zeta w, w ; V) & =(U-\delta F)_{\xi \bar{\xi}}\left(\zeta^{a} w^{a+b}\right) J(\zeta, w)\left|a V_{1}+b \zeta V_{2}\right|^{2} \\
& \geq \mathfrak{L} Q_{\mu}(\zeta w, w ; V) \quad \forall(\zeta, w, V) \in \omega \times \mathbb{C}^{2},
\end{aligned}
$$

for each $\delta: 0<\delta \leq 1$.

Step 3. Completing the proof when the set $\left\{(\zeta, w): w \zeta \neq 0\right.$ and $\left.U_{\xi \bar{\xi}}\left(\zeta^{a} w^{a+b}\right)=0\right\}$ is non-empty

In view of Part $(b)$ of Result [3.2, the definition of $H$ and the estimate (4.3), we have

$$
\begin{aligned}
& \mathfrak{L}(P-\delta H)(\zeta w, w ; V) \geq c_{1}|w|^{2(k-1)}|\zeta|^{\eta-2}\left(\left|V_{1}\right|^{2}+\left|\zeta V_{2}\right|^{2}\right) \\
& \forall(\zeta, w, V) \in \underset{\widetilde{\zeta} \in \overline{D\left(0 ; R_{1}\right)}}{\bigcup}\{\widetilde{\zeta}\} \times \mathbb{T} \times \overline{\mathfrak{C}}_{\beta}(\widetilde{\zeta}),
\end{aligned}
$$

for each $\delta: 0<\delta \leq 1$. Next, from (4.8) and (4.1), we conclude that

$$
\mathfrak{L}(P-\delta H)(\zeta w, w ; V) \geq \mathfrak{L} P(\zeta w, w ; V) \geq 0 \forall(\zeta, w, V) \in \omega \times \mathbb{C}^{2},
$$

for each $\delta: 0<\delta \leq 1$.

Let us stay with the case when $(\triangle U)^{-1}\{0\} \supsetneq\{0\}$ for the moment and complete our argument for this case. It now remains to account for the positivity of the Leviform of $(P-\delta H)$ along vectors that remain unaccounted for by the inequality (4.9). We will have to treat this under separate two cases. In this part of the proof, we shall use the notation $J_{l}(\zeta, w)$ to denote the product $|\zeta|^{\mu-l}|w|^{2(k-1)}, l=0, \ldots, \mu$.

Case $(i) V \in \mathbb{C}^{2}:\left|V_{1}\right| \leq((b / a)-\beta)\left|\zeta V_{2}\right|$ for some $\zeta \in \mathbb{C}$

The above condition implies

$$
\begin{aligned}
\left|a V_{1}+b \zeta V_{2}\right| & \geq b\left|\zeta V_{2}\right|-a\left|V_{1}\right| \geq a \beta\left|\zeta V_{2}\right|, \\
\left|V_{1}\right|^{2}+\left|\zeta V_{2}\right|^{2} & \leq\left((b / a)^{2}+1\right)\left|\zeta V_{2}\right|^{2} .
\end{aligned}
$$


It sufficies to study $\mathfrak{L}(P-\delta H)$ for

$$
\begin{aligned}
&(\zeta, w, V) \in\left\{(\zeta, w, V) \in \omega^{C} \times \mathbb{C}^{2}:|\zeta|<1 / 2,\left|V_{1}\right| \leq((b / a)-\beta)\left|\zeta V_{2}\right|\right\} \\
&:=W_{1}(s=1 / 2) .
\end{aligned}
$$

We appeal to the observation (4.7), which, in conjunction with Part (c) of Result 3.2, tells us that there exist constants $C, M>0$ such that (exploiting the two inequalities above) we may estimate:

$$
\begin{aligned}
\mathfrak{L}(P-\delta H)(\zeta w, w ; V) \geq & C(a \beta)^{2} J_{0}(\zeta, w)\left|V_{2}\right|^{2}-M J_{1}(\zeta, w)\left(\left|V_{1}\right|^{2}+\left|\zeta V_{2}\right|^{2}\right) \\
\geq & J_{0}(\zeta, w)\left[C(a \beta)^{2}-M|\zeta|\left((b / a)^{2}+1\right)\right]\left|V_{2}\right|^{2} . \\
& \forall(\zeta, w, V) \in W_{1}(1 / 2),
\end{aligned}
$$

for each $\delta: 0<\delta \leq 1$. Thus, there exists a positive constant $R_{2} \ll 1$ such that

$$
\begin{aligned}
\mathfrak{L}(P-\delta H)(\zeta w, w ; V) \geq & \frac{C(a \beta)^{2}}{2} J_{0}(\zeta, w)\left|V_{2}\right|^{2} \\
\geq & c_{2} J_{2}(\zeta, w)\left(\left|V_{1}\right|^{2}+\left|\zeta V_{2}\right|^{2}\right) \\
& \forall(\zeta, w, V) \in W_{1}\left(R_{2}\right),
\end{aligned}
$$

for each $\delta: 0<\delta \leq 1$.

Case (ii) $V \in \mathbb{C}^{2}:\left|V_{1}\right| \geq((b / a)+\beta)\left|\zeta V_{2}\right|$ for some $\zeta \in \mathbb{C}$

The inequalities analogous to the ones used in Case $(i)$ are:

$$
\begin{aligned}
\left|a V_{1}+b \zeta V_{2}\right| & \geq a\left|V_{1}\right|-b\left|\zeta V_{2}\right| \geq a \beta((b / a)+\beta)^{-1}\left|V_{1}\right|, \\
\left|V_{1}\right|^{2}+\left|\zeta V_{2}\right|^{2} & \leq\left[((b / a)+\beta)^{-2}+1\right]\left|V_{1}\right|^{2} .
\end{aligned}
$$

It is now evident that the previous argument goes through mutatis mutandis to yield constants $c_{3}, R_{3}>0$ such that

$$
\begin{aligned}
\mathfrak{L}(P-\delta H)(\zeta w, w ; V) \geq & c_{3} J_{2}(\zeta, w)\left(\left|V_{1}\right|^{2}+\left|\zeta V_{2}\right|^{2}\right) \\
& \forall(\zeta, w, V) \in W_{2}\left(R_{3}\right),
\end{aligned}
$$

for each $\delta: 0<\delta \leq 1$, where

$$
W_{2}(s):=\left\{(\zeta, w, V) \in \omega^{\mathrm{C}} \times \mathbb{C}^{2}:|\zeta|<s,\left|V_{1}\right| \geq((b / a)+\beta)\left|\zeta V_{2}\right|\right\} .
$$

From the definition of $H$ given by (4.6) (recall that $(\triangle U)^{-1}\{0\} \supseteq\{0\}$ ), Part $(a)$ is evident. Next; if we define

$$
\sigma:=\min \left\{\varepsilon, R_{1}, R_{2}, R_{3}\right\}
$$

and make the following substitutions

$$
z_{1}=\zeta w, \quad z_{2}=w
$$

then, the statements (4.9), (4.10), (4.11) and (4.12) clearly establish Part $(b)$

Step $\mathbf{3}^{\prime}$. Completing the proof when the set $\left\{(\zeta, w): w \zeta \neq 0\right.$ and $\left.U_{\xi \bar{\xi}}\left(\zeta^{a} w^{a+b}\right)=0\right\}$ is empty

Very similar ideas will work when $(\triangle U)^{-1}\{0\}=\{0\}$. In this situation, Step 2 is irrelevant. Instead, we begin by defining $\gamma:=\inf _{\theta \in[0,2 \pi]} U_{\bar{\xi} \bar{\xi}}\left(e^{i \theta}\right)$. Note that $\gamma>0$. In this case, we define (recall that $U$ is homogeneous of degree $2 m$ )

$$
H\left(z_{1}, z_{2}\right):=\frac{\gamma}{2 m^{2}}\left|z_{1}^{a} z_{2}^{b}\right|^{2 m} \forall\left(z_{1}, z_{2}\right) \in \mathbb{C}^{2} .
$$


In this situation, we can repeat the entire argument given in Step 3 - relying, once again, on the two inequalities that follow the headings "Case $(i)$ " and "Case $(i i)$ " - making the following replacements:

- Replace the definition of $W_{1}(s)$ given in Case $(i)$ by

$$
W_{1}(s):=\left\{(\zeta, w, V) \in \mathbb{C}^{2} \times \mathbb{C}^{2}:|\zeta|<s,\left|V_{1}\right| \leq((b / a)-\beta)\left|\zeta V_{2}\right|\right\} ;
$$

- Replace the definition of $W_{2}(s)$ given in Case $(i i)$ by

$$
W_{2}(s):=\left\{(\zeta, w, V) \in \mathbb{C}^{2} \times \mathbb{C}^{2}:|\zeta|<s,\left|V_{1}\right| \geq((b / a)+\beta)\left|\zeta V_{2}\right|\right\} ;
$$

- Replace the constant $C$, wherever it occurs in Step 3, by the constant $\gamma / 2$.

It is easy to see that this exercise leads us to the following conclusion:

When $(\triangle U)^{-1}\{0\}=\{0\}$, analogues of the estimates (4.11) and (4.12)

are achieved with $H$ as redefined in (4.13) (with the newly-defined $W_{j}(s), j=1,2$ ),

from which the desired result follows for the case $(\triangle U)^{-1}\{0\}=\{0\}$.

\section{THE KEY PROPOSITION}

We are now in a position to prove the key technical proposition of this work. We ought to indicate that the work in this section represents a continuation of the work done in [2. While the philosophy of the proof of Proposition 5.3 below is not very different from the proof of [2, Theorem 1], the estimates needed for Theorem [2.5] affect the details of the proof below. For instance: the proof of Proposition [5.3 would not work without Proposition 4.2. The similarity with [2, Theorem 1] is further visible in our use of the other crucial idea needed in our proof. This result is derived from [11].

Result 5.1 (Version of Prop. 4.1 in [11]). Let $P$ be a plurisubharmonic polynomial on $\mathbb{C}^{n}$ that is homogeneous of degree $2 k$. Let $\omega_{0}$ be a connected component of $\omega(P) \backslash\{0\}$ having the following two properties:

a) There exist closed cones $\overline{\mathcal{K}}_{1}$ and $\overline{\mathcal{K}}_{2}$ such that

$$
\omega_{0} \subset \operatorname{int}\left(\overline{\mathcal{K}}_{1}\right) \subset \overline{\mathcal{K}}_{1} \backslash\{0\} \varsubsetneqq \operatorname{int}\left(\overline{\mathcal{K}}_{2}\right),
$$

and such that $\overline{\mathcal{K}}_{2} \bigcap\left(\omega(P) \backslash \overline{\omega_{0}}\right)=\varnothing$.

b) $\omega_{0}$ does not contain any complex-analytic subvarieties of positive dimension along which $P$ is harmonic.

Then, there exist a smooth function $H \geq 0$ that is homogeneous of degree $2 k$ and constants $C, \varepsilon_{0}>0$, which depend only on $P$, such that $\operatorname{int}\left(\overline{\mathcal{K}}_{2}\right)=\{H>0\}$ and such that for each $\varepsilon: 0<\varepsilon \leq \varepsilon_{0}, \mathfrak{L}(P-\varepsilon H)(z ; v) \geq C \varepsilon\|z\|^{2(k-1)}\|v\|^{2} \forall(z, v) \in \overline{\mathcal{K}}_{2} \times \mathbb{C}^{n}$.

Remark 5.2. The above result is not stated in precisely these words in [11, Proposition 4.1]. The proof of the latter was derived from a construction pioneered by Diederich and Fornaess in [6]. A careful comparison of the proof of [11, Proposition 4.1] with the Diederich-Fornaess construction - keeping in mind the assumption of homogeneity in Result 5.1 - easily reveals that the assumption $(a)$ in Result [5.1 is enough to obtain the above "localised" version of [11, Proposition 4.1]. 
Proposition 5.3. Let $\mathcal{U}$ be a neighbourhood of $0 \in \mathbb{C}^{3}$ and let $\mathcal{V}$ be such that $\{0\} \times \mathcal{V}=\mathcal{U} \cap\left(\{0\} \times \mathbb{C}^{2}\right)$. Let $R \in \mathcal{C}^{\infty}(\mathcal{V}) \bigcap \operatorname{psh}(\mathcal{V})$ and be such that the domain $\Omega_{R}:=\{(w, z) \in \mathcal{U}: \mathfrak{R e} w+R(z)<0\}$ is of finite type. Let

$$
\sum_{n=2 k}^{\infty} P_{n, R}\left(z_{1}, z_{2}\right)
$$

denote the Taylor expansion of $R$ around $z=0$, where $k \geq 2$, and each $P_{n, R}$ is the sum of all monomials having total degree $n$. Set $P:=P_{2 k, R}$. Assume that $P$ has no pluriharmonic terms and has the properties stated in Definition 2.2. If $\mathcal{E}(P) \neq \varnothing$, then:

1) $\mathcal{E}(P)$ is a finite collection of complex lines $L_{1}, \ldots L_{N}$ passing through the origin.

2) For any $x \in L_{j}$, set $[j, x]^{\perp}:=\left(x+L_{j}^{\perp}\right), j=1, \ldots, N$, where the orthogonal complement is taken with respect to the standard inner product on $\mathbb{C}^{2}$. Assume that for each $x \in L_{j} \backslash\{0\}$

$$
\operatorname{Ord}\left(\left.P\right|_{[j, x]^{\perp}}-P(x), x\right) \leq \operatorname{Ord}\left(\left.R\right|_{[j, x]^{\perp}}-R(x), x\right),
$$

and let $\mu_{j}$ denote the generic value on $L_{j}$ of the left-hand side of (5.1), $j=$ $1, \ldots, N$. Then, there is an algebraic change of coordinate centered at $0 \in \mathbb{C}^{3}$ and a positive integer $D$, chosen suitably so as to give us $(a)-(d)$ below, such that if $\left(W, Z_{1}, Z_{2}\right)$ denotes the new coordinates, then $Z=z$; and if $\Omega_{R}=\{(W, z): \mathfrak{R e} W+\rho(z)<0\}$ is the representation of $\Omega_{R}$ relative to the new coordinates, then the associated $P_{n, \rho}$ contain no pluriharmonic terms for $n=2 k, \ldots, D$, and $P_{2 k, R}=P_{2 k, \rho}=P$. Furthermore, there exist constants $C, \delta_{0}, r_{0}>0$; a non-negative function $H \in \mathcal{C}^{\infty}\left(\mathbb{C}^{2} \backslash\{0\}\right) \cap \mathcal{C}^{2}\left(\mathbb{C}^{2}\right)$ that is homogeneous of degree $2 k$; closed cones $\overline{\mathcal{K}}_{j}^{1}$ and $\overline{\mathcal{K}}_{j}^{2}$ satisfying

$$
\overline{\mathcal{K}}_{j}^{1} \backslash\{0\} \varsubsetneqq \operatorname{int}\left(\overline{\mathcal{K}}_{j}^{2}\right), \quad j=1, \ldots, N ;
$$

and, for each $\delta \in\left(0, \delta_{0}\right)$, a constant $r_{\delta}>0$; subharmonic functions $U_{j, \delta} \in$ $\mathcal{C}^{\infty}(\mathbb{C} \backslash\{0\}) \cap \mathcal{C}^{2}(\mathbb{C})$ that are homogeneous of degree $2 M_{j}, j=1, \ldots, N$, and are strictly subharmonic away from $0 \in \mathbb{C} ;$ and a function $G_{\delta} \in \mathcal{C}^{\infty}\left(\mathbb{C}^{2}\right) \cap \operatorname{psh}\left(\mathbb{C}^{2}\right)$ such that:

a) $G_{\delta}$ is strictly plurisubharmonic on $\mathbb{C}^{2} \backslash\{0\}$.

b) For each $j \leq N, G_{\delta}(z)=(P-\delta H)(z)+U_{j, \delta}\left(z_{k(j)}\right) \quad \forall\left(z_{1}, z_{2}\right) \in$ $\overline{\mathcal{K}}_{j}^{1} \cap \mathbb{B}^{2}\left(0 ; r_{0}\right)$, where $k(j)=1$ or 2 depending on $j=1, \ldots, N$.

c) $G_{\delta}\left(z_{1}, z_{2}\right)=(P-\delta H)\left(z_{1}, z_{2}\right) \forall\left(z_{1}, z_{2}\right) \in\left(\mathbb{C}^{2} \backslash \cup_{j=1}^{N} \overline{\mathcal{K}}_{j}^{2}\right) \cap \mathbb{B}^{2}\left(0 ; r_{0}\right)$.

d) For each $j \leq N$ such that the complex line $L_{j}$ has the form $L_{j}=\{z$ : $\left.z_{1}=\xi_{j} z_{2}\right\}, \xi_{j} \in \mathbb{C}$,

$$
\begin{aligned}
&\left(G_{\delta}-\rho\right)(z) \leq-\delta C\left(\left|z_{1}-\xi_{j} z_{2}\right|^{\mu_{j}}\left|z_{2}\right|^{2 k-\mu_{j}}+\left|z_{2}\right|^{2 M_{j}}\right) \\
& \forall\left(z_{1}, z_{2}\right) \in \overline{\mathcal{K}}_{j}^{1} \bigcap \mathbb{B}^{2}\left(0 ; r_{\delta}\right) .
\end{aligned}
$$

If $L_{j}=\left\{z: z_{2}=0\right\}$, then the above inequality holds with the positions of $z_{1}$ and $z_{2}$ swapped and $\xi_{j}=0$.

e) $\left(G_{\delta}-\rho\right)(z) \leq-\delta C\|z\|^{2 k} \forall\left(z_{1}, z_{2}\right) \in\left(\mathbb{C}^{2} \backslash \cup_{j=1}^{N} \overline{\mathcal{K}}_{j}^{1}\right) \cap \mathbb{B}^{2}\left(0 ; r_{\delta}\right)$. 
Proof. Note that Part (1) follows simply from Proposition 3.1. Let $\overline{\mathcal{K}}$ be the closed cone with the properties given in Definition 2.2 whose existence is guaranteed by hypothesis. By assumption, we can find a slightly larger cone $\overline{\mathcal{K}}_{*}$ such that

$$
\omega(P) \backslash\left(\bigcup_{j=1}^{N} L_{j}\right) \subset \operatorname{int}(\overline{\mathcal{K}}) \subset \overline{\mathcal{K}} \backslash\{0\} \varsubsetneqq \operatorname{int}\left(\overline{\mathcal{K}}_{*}\right),
$$

and such that $\overline{\mathcal{K}}_{*} \bigcap\left(\bigcup_{j=1}^{N} L_{j}\right)=\{0\}$.

Step 1. Constructing $G_{\delta}$

In view of the assumption that $P$ has no pluriharmonic terms, $\left.P\right|_{L_{j}} \equiv 0$. Please note: in the interests of brevity, our arguments below will be framed for complex lines in $\mathcal{E}(P)$ of the form

$$
L_{j}=\left\{z: z_{1}=\xi_{j} z_{2}\right\}, \quad \xi_{j} \in \mathbb{C},
$$

only. If $\mathcal{E}(P) \ni\left\{z: z_{2}=0\right\}=: L_{j}$, then a separate argument will be provided for a statement relating to $L_{j}$ only if that statement does not follow mutatis mutandis from the arguments given. In particular: for the purposes of Step 1, we may assume that $L_{j}=\left\{z: z_{1}=\xi_{j} z_{2}\right\}, j=1, \ldots, N$. The hypotheses of Proposition 4.2 are satisfied by

$$
\widetilde{j P}\left(z_{1}, z_{2}\right):=P\left(z_{1}+\xi_{j} z_{2}, z_{2}\right) .
$$

Hence, we can find:

- a constant $c_{1}>0$ that depends only on $P$;

- constants $\sigma_{j}>0, j=1, \ldots, N$, that depend only on $P$ and $j$;

- functions $H_{j} \in \mathcal{C}^{\infty}\left(\mathbb{C}^{2} \backslash\{0\}\right) \cap \mathcal{C}^{2}\left(\mathbb{C}^{2}\right)$ that are homogeneous of degree $2 k$; and

- a positive decreasing function $B:(0,1) \longrightarrow \mathbb{R}$;

such that

$$
\begin{aligned}
\mathfrak{L}\left(P-\delta H_{j}\right)\left(z ;\left(V_{1}, V_{2}\right)\right) \geq & B(t)\|z\|^{2(k-1)}\|V\|^{2} \\
& \forall z: t \sigma_{j}\left|z_{2}\right|<\left|z_{1}-\xi_{j} z_{2}\right|<\sigma_{j}\left|z_{2}\right|, \quad \forall V \in \mathbb{C}^{2}, \\
H_{j}\left(z_{1}, z_{2}\right)> & c_{1}\left|z_{1}-\xi_{j} z_{2}\right|^{\mu_{j}}\left|z_{2}\right|^{2 k-\mu_{j}} \quad \forall z \in \mathcal{K}\left(\xi_{j} ; \sigma_{j}\right) \backslash L_{j},
\end{aligned}
$$

for $t \in(0,1), j=1, \ldots, N$, and for $\delta \in(0,1]$. Recall that $\mu_{j} \in \mathbb{Z}_{+}$are as determined by the condition (5.1).

Note, furthermore, that, by the finite-type assumption on $\Omega_{R}$, if we define

$$
M_{j}:=\frac{\inf \left\{n \in \mathbb{Z}_{+}:\left.P_{n, R}\right|_{L_{j}} \text { is non-harmonic on } L_{j}\right\}}{2},
$$

then $M_{j}<\infty \forall j=1, \ldots, N$. Let us now write $D:=\max _{j \leq N} 2 M_{j}$. It is a wellknown trick that we can make a global holomorphic change of coordinates and work with new coordinates $\left(W, Z_{1}, Z_{2}\right)$ having the form

$$
\begin{aligned}
W & =w-\left(\text { a holomorphic polynomial in } z_{1} \text { and } z_{2} \text { of degree } \leq D\right), \\
Z & =z,
\end{aligned}
$$

such that if the representation of $\Omega_{R}$ with respect to these coordinates is

$$
\Omega_{R}=\{(W, Z): \mathfrak{R e} W+\rho(Z)<0\},
$$

then the Taylor expansion of $\rho$ around $Z=0$ contains no pluriharmonic terms of degree $\leq D$, and $P_{2 k, R}=P_{2 k, \rho}$. To clarify: the holomorphic polynomial that is a part of the definition of $(W, Z)$ is the sum of all monomials in $z_{1}$ and $z_{2}$ whose real 
and imaginary parts are pluriharmonic terms in $P_{n, R}, n=2 k+1, \ldots, D$. Let us call this polynomial $\Theta$. Now, owing to the property (5.4) of $\left(W, Z_{1}, Z_{2}\right)$ :

- The condition (5.1) remains unchanged when $R$ is replaced by $\rho$. To understand this, first note that the left-hand side of (5.1) remains unchanged. Now, suppose $L_{j}=\operatorname{span}_{\mathbb{C}}\left\{\left(B_{1}, B_{2}\right)\right\}$. If $\mathbf{O}_{x, j} \in[1, \infty]$ denotes the degree of the first non-zero monomial in $\tau$ and $\bar{\tau}$ of the Taylor expansion in $(\tau, \bar{\tau})$ (around $\tau=0)$ of $\left(R\left(x+\tau\left(B_{2},-B_{1}\right)\right)-R(x)\right)$, then (5.1) states that

$$
\mathbf{O}_{x, j} \geq \operatorname{Ord}\left(\left.P\right|_{[j, x]^{\perp}}-P(x), x\right) \text {. }
$$

Now, as the monomials of the polynomial $\left(\Theta\left(x+\tau\left(B_{2},-B_{1}\right)\right)-\Theta(x)\right)$ occur in the aforementioned Taylor expansion, the degree of the first non-zero monomial in $\tau$ and $\bar{\tau}$ of the Taylor expansion in $(\tau, \bar{\tau})$ of $\left(\rho\left(x+\tau\left(B_{2},-B_{1}\right)\right)-\right.$ $\rho(x))$ cannot be less than $\mathbf{O}_{x, j}$. Hence our claim.

- None of the assertions made thus far in this proof are affected.

From this point, we shall work with the defining function $(\mathfrak{R e} W+\rho(z))$, and we shall simply write $P_{n, \rho}=: P_{n}, n \in \mathbb{Z}_{+}$. It follows from an argument very similar to the one used in the proof of Lemma 3.4 that the functions

$$
u_{j}(s):=P_{2 M_{j}}\left(\xi_{j} s, s\right), \quad s \in \mathbb{C},
$$

$j=1, \ldots, N$, are subharmonic, non-harmonic functions. In particular, therefore, the $M_{j}$ 's defined above are integers. We invoke Result 3.2 to obtain:

- constants $c_{2}, B_{2}>0$ that depend only on $\rho$; and

- functions $h_{j} \in \mathcal{C}^{\infty}(\mathbb{C} \backslash\{0\}) \cap \mathcal{C}^{2}(\mathbb{C})$ that are homogeneous of degree $2 M_{j}, j=$ $1, \ldots, N$

such that

$$
\begin{aligned}
h_{j}(s) & \geq c_{2}|s|^{2 M_{j}} \forall s \in \mathbb{C}, \\
\partial_{s \bar{s}}^{2}\left(u_{j}-\delta h_{j}\right)(s) & \geq \delta B_{2}|s|^{2\left(M_{j}-1\right)} \forall s \in \mathbb{C}, \forall \delta: 0<\delta \leq 1,
\end{aligned}
$$

$j=1, \ldots, N$. Lastly, in view of Result [5.1, we can find a smooth function $H_{0} \geq 0$ that is homogeneous of degree $2 k$, and constants $B_{3}, \varepsilon_{0}>0$ such that

$$
\begin{aligned}
& \left\{z: H_{0}>0\right\}=\operatorname{int}\left(\overline{\mathcal{K}}_{*}\right), \\
& \qquad \begin{aligned}
\mathfrak{L}\left(P-\delta H_{0}\right) & (z ; V) \\
& \geq \delta B_{3}\|z\|^{2(k-1)}\|V\|^{2} \quad \forall(z, v) \in \overline{\mathcal{K}}_{*} \times \mathbb{C}^{2}, \text { and } \forall \delta \in\left(0, \varepsilon_{0}\right) .
\end{aligned}
\end{aligned}
$$

Let $\alpha>0$ be so small that

$$
\begin{aligned}
2 \alpha & \leq \sigma_{j}, \quad j=1, \ldots, N, \\
\left.\left(\overline{\mathcal{K}\left(\xi_{j}\right.} ; 2 \alpha\right) \cap S^{3}\right) \bigcap\left(\overline{\mathcal{K}}_{*} \cap S^{3}\right) & =\varnothing \quad \forall j \leq N, \\
\left.\left.\left(\overline{\mathcal{K}\left(\xi_{j}\right.} ; 2 \alpha\right) \cap S^{3}\right) \bigcap\left(\overline{\mathcal{K}\left(\xi_{k}\right.} ; 2 \alpha\right) \cap S^{3}\right) & =\varnothing \quad \text { if } j \neq k .
\end{aligned}
$$

Here, $S^{3}$ denotes the unit sphere in $\mathbb{C}^{2}$. The parameter $\alpha$ will be used to define the cones $\overline{\mathcal{K}}_{j}^{1}$ and $\overline{\mathcal{K}}_{j}^{2}, j=1, \ldots, N$. Let us now define

$$
\left.\left.V_{j}:=\overline{\mathcal{K}\left(\xi_{j}\right.} ; \alpha\right) \cap S^{3}, \text { and } W_{j}:=\overline{\mathcal{K}\left(\xi_{j}\right.} ; 2 \alpha\right) \cap S^{3} .
$$

Let $\chi_{j}: S^{3} \longrightarrow[0,1]$ be a smooth cut-off function such that $\left.\chi_{j}\right|_{V_{j}} \equiv 1$ and $\operatorname{supp}\left(\chi_{j}\right) \subset W_{j}, j=1, \ldots, N$. Let us define $\Psi_{j}(z):=\chi_{j}(z /\|z\|) \forall z \in \mathbb{C}^{2} \backslash\{0\}$. Finally, if $\Phi: \mathbb{C}^{2} \longrightarrow \mathbb{R}$ is a function that is homogeneous of degree $d>0$, then 
we shall abuse notation somewhat and use the expression $\Psi_{j}(z) \Phi(z)$ as having the following meaning:

$$
\Psi_{j}(z) \Phi(z):= \begin{cases}\Psi_{j}(z) \Phi(z), & \text { if } z \neq 0 \\ 0, & \text { if } z=0 .\end{cases}
$$

Note that $\Psi_{j} \Phi$ has the same regularity as $\Phi$ and is homogeneous of degree $d$. Before we define $G_{\delta}$, let us define a preliminary function $\widetilde{G}_{\delta}$ :

$$
\widetilde{G}_{\delta}(z):=P(z)-\delta H_{0}(z)+\sum_{j=1}^{N}\left[u_{j}\left(z_{2}\right)-\delta\left(H_{j}(z)+h_{j}\left(z_{2}\right)\right)\right] \Psi_{j}(z) \forall z \in \mathbb{C}^{2} .
$$

Note that if $\mathcal{E}(P) \ni\left\{z: z_{2}=0\right\}=: L_{j^{0}}$, then the sum on the right-hand side above would contain the summand $\left[u_{j^{0}}\left(z_{1}\right)-\delta\left(H_{j^{0}}(z)+h_{j^{0}}\left(z_{1}\right)\right)\right] \Psi_{j^{0}}(z)$. Also note that, as $\Psi_{j} \equiv 1$ on $\left.\overline{\mathcal{K}\left(\xi_{j}\right.} ; \alpha\right) \bigcap S^{3}$, Proposition 4.2 and (5.6) imply that $\widetilde{G}_{\delta}$ is strictly plurisubharmonic on $\left.\overline{\mathcal{K}\left(\xi_{j}\right.} ; \alpha\right) \backslash\{0\}, j=1, \ldots, N$. But, since strict plurisubharmonicity is an open condition, we infer from continuity and homogeneity that

(i) $\exists \varepsilon \ll 1$ such that $\widetilde{G}_{\delta}$ is strictly plurisubharmonic on $\mathcal{K}\left(\xi_{j} ; \alpha+\varepsilon\right)$; for each $j=1, \ldots, N$, and for each $\delta: 0<\delta \leq 1 / 2$. In view of $(i)$ above we need to examine the Levi-form of $\widetilde{G}_{\delta}$ on $\left.\mathcal{K}\left(\xi_{j} ; 2 \alpha\right) \backslash \overline{\mathcal{K}\left(\xi_{j}\right.} ; \alpha+\varepsilon\right)$. By our definition of the $\Psi_{j}$ 's, we can find a $\beta>0$ such that

$$
\left.\left(1-\Psi_{j}\right)(z) \geq \beta \quad \forall z \in \mathcal{K}\left(\xi_{j} ; 2 \alpha\right) \backslash \overline{\mathcal{K}\left(\xi_{j}\right.} ; \alpha+\varepsilon\right), \quad j=1, \ldots, N .
$$

Furthermore, an application of (5.2), and an appeal to the properties of $P$ stated in Definition 2.2, respectively, imply that there exists a constant $\gamma>0$ such that

$\left.z \in\left(\mathcal{K}\left(\xi_{j} ; 2 \alpha\right) \backslash \overline{\mathcal{K}\left(\xi_{j}\right.} ; \alpha+\varepsilon\right)\right) \Rightarrow\left\{\begin{aligned} \mathfrak{L}\left(P+u_{j}-\delta\left(H_{j}+h_{j}\right)\right)(z ; V) & \geq 0, \\ \mathfrak{L} P(z ; V) & \geq \gamma\|z\|^{2(k-1)}\|V\|^{2},\end{aligned}\right.$

for each $V \in \mathbb{C}^{2}$ and each $j=1, \ldots, N$, and for each $\delta: 0<\delta \leq 1 / 2$. For each $j=1, \ldots, N$, let us write $F_{j}\left(z_{1}, z_{2}\right):=H_{j}\left(z_{1}, z_{2}\right)+h_{j}\left(z_{2}\right)$. From the last three inequalities, we can estimate:

$$
\begin{gathered}
\mathfrak{L} \widetilde{G}_{\delta}(z ; V) \geq\left(1-\Psi_{j}\right)(z) \mathfrak{L} P(z ; V)+\left(u_{j}\left(z_{2}\right)-\delta F_{j}(z)\right) \mathfrak{L} \Psi_{j}(z ; V) \\
+2 \mathfrak{R e}\left[\sum_{\mu, \nu \leq 2} \partial_{\mu} \Psi_{j}(z) \partial_{\bar{\nu}}\left(u_{j}-\delta F_{j}\right)(z) V_{\mu} \overline{V_{\nu}}\right] \\
\geq \beta \gamma\|z\|^{2(k-1)}\|V\|^{2}-\delta S_{1}(z ; V)-S_{2}(z ; V) \\
\left.\forall z \in \mathcal{K}\left(\xi_{j} ; 2 \alpha\right) \backslash \overline{\mathcal{K}\left(\xi_{j}\right.} ; \alpha+\varepsilon\right) \text { and } \forall V \in \mathbb{C}^{2},
\end{gathered}
$$

where $S_{1}(z ; V)$ and $S_{2}(z ; V)$ are as follows. We first consider $S_{1}(z ; V)$, in which case we can find a large constant $K_{1}>0$ such that

$$
\begin{aligned}
S_{1}(z ; V) & :=2 \sum_{\mu, \nu \leq 2}\left|\partial_{\mu} \Psi_{j}(z) \partial_{\bar{\nu}} H_{j}(z) V_{\mu} \overline{V_{\nu}}\right|+H_{j}(z)\left|\mathfrak{L} \Psi_{j}(z ; V)\right| \\
& \left.\leq K_{1}\|z\|^{2(k-1)}\|V\|^{2} \quad \forall(z, V) \in\left(\mathcal{K}\left(\xi_{j} ; 2 \alpha\right) \backslash \overline{\mathcal{K}\left(\xi_{j}\right.} ; \alpha+\varepsilon\right)\right) \times \mathbb{C}^{2},
\end{aligned}
$$

for each $j=1, \ldots, N$. In a similar way, we can find a large constant $K_{2}>0$ such that

$$
\left.S_{2}(z ; V) \leq K_{2}\left|z_{2}\right|^{2\left(M_{j}-1\right)}\|V\|^{2} \quad \forall(z, V) \in\left(\mathcal{K}\left(\xi_{j} ; 2 \alpha\right) \backslash \overline{\mathcal{K}\left(\xi_{j}\right.} ; \alpha+\varepsilon\right)\right) \times \mathbb{C}^{2},
$$


for each $j=1, \ldots, N$. Let $\delta^{*}>0$ be so small that $\delta K_{1} \leq \beta \gamma / 4 \forall \delta \in\left(0, \delta^{*}\right]$. Then, we can find an $r_{0}>0$ such that, in view of (5.11) and (5.12), the following holds:

$$
\begin{gathered}
\mathfrak{L} \widetilde{G}_{\delta}(z ; V) \geq\left(\beta \gamma-\delta K_{1}\right)\|z\|^{2(k-1)}-K_{2}\left|z_{2}\right|^{2\left(M_{j}-1\right)}\|V\|^{2} \\
\geq \frac{\beta \gamma}{2}\|z\|^{2(k-1)}\|V\|^{2} \\
\left.\forall z \in\left(\mathcal{K}\left(\xi_{j} ; 2 \alpha\right) \backslash \overline{\mathcal{K}\left(\xi_{j}\right.} ; \alpha+\varepsilon\right)\right) \bigcap \mathbb{B}^{2}\left(0 ; 2 r_{0}\right), \\
\forall V \in \mathbb{C}^{2}, \text { and } \forall \delta: 0<\delta \leq \delta^{*},
\end{gathered}
$$

for each $j=1, \ldots, N$. Let us now set

$$
\begin{aligned}
\widetilde{H} & :=H_{0}+\sum_{j=1}^{N} \Psi_{j} H_{j}, \\
\delta_{0} & :=\min \left(\varepsilon_{0}, \delta^{*}\right) .
\end{aligned}
$$

So far, in view of $(i)$ above and the bound (5.13) , we have accomplished the following:

(ii) $\widetilde{G}_{\delta}$ is strictly plurisubharmonic on $\mathbb{B}^{2}\left(0 ; 2 r_{0}\right) \backslash\{0\} \forall \delta \in\left(0, \delta_{0}\right)$.

(iii) $\{z: \widetilde{H}>0\}=\operatorname{int}\left(\overline{\mathcal{K}}_{*}\right) \bigcup\left(\bigcup_{j=1}^{N}\left(\mathcal{K}\left(\xi_{j} ; 2 \alpha\right) \backslash L_{j}\right)\right)$.

We carry out the following three steps to transform $\widetilde{G}_{\delta}$ to $G_{\delta}$ :

A) We make a small perturbation of $\widetilde{H}$ to obtain a function $H$ having the same regularity as $\widetilde{H}$ and homogeneous of degree $2 k$ such that $H^{-1}\{0\}=\bigcup_{j=1}^{N} L_{j}$.

B) Ensure that the perturbation in (A) is so small that if we set

$$
\widetilde{\Gamma}_{\delta}(z):=P(z)-\delta H(z)+\sum_{j=1}^{N}\left(u_{j}\left(z_{2}\right)-\delta h_{j}\left(z_{2}\right)\right) \Psi_{j}(z) \forall z \in \mathbb{C}^{2},
$$

then $\widetilde{\Gamma}_{\delta}$ is strictly plurisubharmonic on $\mathbb{B}^{2}\left(0 ; 2 r_{0}\right) \backslash\{0\} \forall \delta \in\left(0, \delta_{0}\right)$. The process of achieving this has been described in the proof of [2, Theorem 1] and, hence, we shall not repeat this argument.

C) Since $\widetilde{\Gamma}_{\delta}$ is strictly plurisubharmonic on $\mathbb{B}^{2}\left(0 ; 2 r_{0}\right) \backslash\{0\}$, it is a well-known fact that we can extend $\widetilde{\Gamma}_{\delta}$ to a function $G_{\delta}$ on $\mathbb{C}^{2}$ that is strictly plurisubharmonic on $\mathbb{C}^{2} \backslash\{0\}$ such that

$$
\left.\widetilde{\Gamma}_{\delta}\right|_{\mathbb{B}^{2}\left(0 ; r_{0}\right)}=\left.G_{\delta}\right|_{\mathbb{B}^{2}\left(0 ; r_{0}\right)} \quad \text { for each } \delta \in\left(0, \delta_{0}\right)
$$

From (C) above, Part 2a) of this proposition follows. If we define:

$$
\begin{aligned}
\overline{\mathcal{K}}_{j}^{k} & \left.:=\overline{\mathcal{K}\left(\xi_{j}\right.} ; k \alpha\right), \quad k=1,2, \\
U_{j, \delta} & :=u_{j}-\delta h_{j},
\end{aligned}
$$

for each $j=1, \ldots, N$, then, in view of (5.14) and (5.15), Parts 2b) and 2c) also follow. The next step of this proof is to shrink $r_{0}$, to the extent necessary, to obtain an $r_{\delta}>0$ so that Parts $2 d$ ) and $2 e$ ) follow.

Step 2. Estimates on the size of $\left(G_{\delta}-\rho\right)$

For the same reasons as described in Step 1, we shall argue as though each $L_{j}$ is of the form $\left\{z: z_{1}=\xi_{j} z_{2}\right\}$ for some $\xi_{j} \in \mathbb{C}$. Analogous arguments will follow if, for 
some $j^{0} \leq N, L_{j^{0}}=\left\{z: z_{2}=0\right\}$. So, let us fix a $j \leq N$ and write

$$
\begin{aligned}
P_{n}(z) & =P_{n}\left(\left(z_{1}-\xi_{j} z_{2}\right)+\xi_{j} z_{2}, z_{2}\right) \\
& \equiv \sum_{|\overline{\boldsymbol{\eta}}|+|\overline{\boldsymbol{\nu}}|=n} C_{\overline{\boldsymbol{\eta}} \boldsymbol{\nu}}(j, n)\left(z_{1}-\xi_{j} z_{2}\right)^{\eta_{1}}\left(\bar{z}_{1}-\bar{\xi}_{j} \bar{z}_{2}\right)^{\eta_{2}} z_{2}^{\nu_{1}} \bar{z}^{\nu_{2}},
\end{aligned}
$$

for each $n=2 k+1, \ldots, 2 M_{j}$, and $j=1, \ldots, N$. Here $\overline{\boldsymbol{\eta}}$ and $\overline{\boldsymbol{\nu}}$ denote multi-indices. We clarify some notation

$$
\begin{array}{rlrl}
\overline{\boldsymbol{\eta}} & =\left(\eta_{1}, \eta_{2}\right), & & |\overline{\boldsymbol{\eta}}|=\eta_{1}+\eta_{2}=: \eta, \\
\overline{\boldsymbol{\nu}} & =\left(\nu_{1}, \nu_{2}\right), & & |\overline{\boldsymbol{\nu}}|=\nu_{1}+\nu_{2}=: \nu, \\
\mathfrak{s u p p}(j, n) & =\left\{\left(\eta_{1}, \eta_{2}, \nu_{1}, \nu_{2}\right) \in \mathbb{N}^{4}: C_{\overline{\boldsymbol{\eta}}}(j, n) \neq 0\right\} .
\end{array}
$$

Recall that, owing to the change of coordinate described in Step 1, $\left.P_{n}\right|_{L_{j}} \equiv 0$ for $n=2 k+1, \ldots, 2 M_{j}-1$. Thus, we can conclude the following facts:

- For $n=2 k+1, \ldots, 2 M_{j}-1$, the condition (5.1) tells us that $\left(\eta_{1}, \eta_{2}, \nu_{1}, \nu_{2}\right) \in$ $\mathfrak{s u p p}(j, n) \Longrightarrow \eta \geq \mu_{j}$.

- $u_{j}(s)=\sum_{(0, \overline{\boldsymbol{\nu}}) \in \mathfrak{s u p p}\left(j, 2 M_{j}\right)} C_{0 \overline{\boldsymbol{\nu}}}\left(j, 2 M_{j}\right) s^{\nu_{1}} \overline{\boldsymbol{s}}^{\nu_{2}}$.

Hence, we get the following estimate

$$
\begin{aligned}
(\overline{\boldsymbol{\eta}}, \overline{\boldsymbol{\nu}}) \in & \mathfrak{s u p p}(j, n), 2 k+1 \leq n \leq 2 M_{j} \text { and } \eta \geq 1 \\
\Longrightarrow & \left|C_{\overline{\boldsymbol{\eta}}}(j, n)\left(z_{1}-\xi_{j} z_{2}\right)^{\eta_{1}}\left(\bar{z}_{1}-\bar{\xi}_{j} \bar{z}_{2}\right)^{\eta_{2}} z_{2}^{\nu_{1}} \bar{z}^{\nu_{2}}\right| \\
& \left.\leq \alpha^{\eta-\mu_{j}}\left|C_{\overline{\boldsymbol{\eta}}}(j, n)\right|\left|z_{1}-\xi_{j} z_{2}\right|^{\mu_{j}}\left|z_{2}\right|^{n-\mu_{j}} \quad \forall z \in \overline{\mathcal{K}\left(\xi_{j}\right.} ; \alpha\right) .
\end{aligned}
$$

Furthermore, if we write

$$
\rho(z)=\sum_{n=2 k}^{2 M_{j}} P_{n}(z)+\mathcal{R}_{j}(z)
$$

for each $j=1, \ldots, N$, then there exists a constant $K_{3}>0$ such that

$$
\left|\mathcal{R}_{j}(z)\right| \leq K_{3}\|z\|^{2 M_{j}+1} \forall z \in \mathbb{B}^{2}(0 ; 1) .
$$

Then, in view of the estimates (5.3), (5.5), (5.16) and (5.17), there is a large constant $K_{4}>0$ such that

$$
\begin{gathered}
\left(G_{\delta}-\rho\right)(z) \leq-\delta\left(c_{1}\left|z_{1}-\xi_{j} z_{2}\right|^{\mu_{j}}\left|z_{2}\right|^{2 k-\mu_{j}}+c_{2}\left|z_{2}\right|^{2 M_{j}}\right) \\
+K_{4} \sum_{j=2 k+1}^{2 M_{j}}\left|z_{1}-\xi_{j} z_{2}\right|^{\mu_{j}}\left|z_{2}\right|^{n-\mu_{j}}+K_{3}\|z\|^{2 M_{j}+1} \\
\left.\forall z \in \overline{\mathcal{K}\left(\xi_{j}\right.} ; \alpha\right) \bigcap \mathbb{B}^{2}(0 ; 1),
\end{gathered}
$$

for each $j=1, \ldots, N$. Since $n>2 k$ in every occurrence of $n$ in the above inequality, we can find an $r_{\delta}>0$ sufficiently small and a constant $C>0$ (independent of $\delta$ ) such that

$$
\begin{aligned}
&\left(G_{\delta}-\rho\right)(z) \leq-\delta C\left(\left|z_{1}-\xi_{j} z_{2}\right|^{\mu_{j}}\left|z_{2}\right|^{2 k-\mu_{j}}+\left|z_{2}\right|^{2 M_{j}}\right) \\
&\left.\forall\left(z_{1}, z_{2}\right) \in \overline{\mathcal{K}\left(\xi_{j}\right.} ; \alpha\right) \bigcap \mathbb{B}^{2}\left(0 ; r_{\delta}\right),
\end{aligned}
$$

for each $j=1, \ldots, N$ and for each $\delta \in\left(0, \delta_{0}\right)$. This establishes Part $\left.2 d\right)$. As for Part $2 e)$, the recipe given by $(\mathrm{A})-(\mathrm{C})$ above implies that $\left(G_{\delta}-\rho\right)(z)$ is dominated by a strictly negative function that is homogeneous of degree $2 k$ (see [2, pp. 53-54] 
for details) in $\left.\left(\mathbb{C}^{2} \backslash \cup_{j=1}^{N} \overline{\mathcal{K}\left(\xi_{j}\right.} ; \alpha\right)\right)$, provided $\|z\|$ is small. Thus, by arguments very similar to the preceding one, Part $2 e$ ) follows.

\section{The Proof of Theorem 2.5}

We begin by stating that several parts of this proof are based on ideas in the proof of Main Theorem 1 of [2]. Define $\nu:=\operatorname{lcm}\left(m_{1}, m_{2}\right)$ (i.e. the least common multiple of $m_{1}$ and $m_{2}$ ) and write $\sigma_{j}:=\nu / m_{j}, j=1,2$. Next, define the two proper holomorphic maps

$$
\begin{aligned}
\psi: \mathbb{C}^{3} \longrightarrow \mathbb{C}^{3}, \quad \psi\left(s, t_{1}, t_{2}\right) & :=\left(s, t_{1}^{\sigma_{1}}, t_{2}^{\sigma_{2}}\right), \\
\Psi: \mathbb{C}^{2} \longrightarrow \mathbb{C}^{2}, \quad \Psi\left(t_{1}, t_{2}\right) & :=\left(t_{1}^{\sigma_{1}}, t_{2}^{\sigma_{2}}\right) .
\end{aligned}
$$

Finally, write $\varphi:=(P+Q)$ and $R:=\varphi \circ \Psi$. Also, write $D:=\left\{(w, z) \in V_{\zeta}\right.$ : $\mathfrak{R e} w+\varphi(z)<0\}$. The following are easy to verify, and we shall not dwell on the details:

- $R$ is plurisubharmonic.

- Let

$$
\sum_{n=m_{0}}^{\infty} \Pi_{n, R}\left(t_{1}, t_{2}\right)
$$

denote the Taylor expansion of $R$ around $t=0$, with each $\Pi_{n, R}$ being the sum of all monomials having total degree $n$. Then, $m_{0}=\nu$.

- The domain $\psi^{-1}(D)$ is given by

$$
\omega_{R}:=\left\{(s, t) \in \psi^{-1}\left(V_{\zeta}\right): \mathfrak{R e} s+R(t)<0\right\} .
$$

- Write $\Pi:=\Pi_{\nu, R}$. Then, $\Pi$ satisfies all the properties satisfied by $P:=P_{2 k, R}$ of Proposition 5.3 (refer to [2, pp. 59-60] for details).

- Furthermore, the domain $\omega_{R}$ satisfies all the hypotheses of Proposition 5.3.

It would be useful to look more closely at the second assertion. We state the following general fact for later use:

Fact 6.1. Let $\varphi$ be any $\mathcal{C}^{\infty}$-smooth function defined around $0 \in \mathbb{C}^{2}$, and let $R:=$ $\varphi \circ \Psi$, where $\Psi$ is as defined above. Let $\Pi_{n, R}, n \in \mathbb{N}$, be as defined above and let

$$
\sum_{\eta} \mathcal{Q}_{\eta, \varphi}
$$

be a formal rearrangement of the terms of the Taylor expansion of $\varphi$ around $z=0$ such that $\mathcal{Q}_{\eta, \varphi}\left(r^{1 / m_{1}} z_{1}, r^{1 / m_{2}} z_{2}\right)=r^{\eta} \mathcal{Q}_{\eta, \varphi}\left(z_{1}, z_{2}\right) \forall r>0$. If $\Pi_{n, R}=\mathcal{Q}_{\eta, \varphi} \circ \Psi$, then $n=\nu \eta$.

To see this simple fact, we fix a $t \in \mathbb{C}^{2}$ such that $\Pi_{n, R}(t) \neq 0$. Thus, by definition

$$
r^{n} \Pi_{n, R}(t)=\Pi_{n, R}(r t)=\mathcal{Q}_{\eta, \varphi}\left(r^{\sigma_{1}} t_{1}^{\sigma_{1}}, r^{\sigma_{2}} t_{2}^{\sigma_{2}}\right) \forall r>0 .
$$

On the other hand

$$
\mathcal{Q}_{\eta, \varphi}\left(r^{\sigma_{1}} t_{1}^{\sigma_{1}}, r^{\sigma_{2}} t_{2}^{\sigma_{2}}\right)=\mathcal{Q}_{\eta, \varphi}\left(\left(r^{\nu}\right)^{1 / m_{1}} t_{1}^{\sigma_{1}},\left(r^{\nu}\right)^{1 / m_{2}} t_{2}^{\sigma_{2}}\right)=r^{\nu \eta} \mathcal{Q}_{\eta, \varphi} \circ \Psi(t) \forall r>0 .
$$

Comparing the two equations above, we conclude that $n=\nu \eta$. The second assertion above is just a special case of Fact 6.1.

As in the proof of Proposition 5.3, in the interests of brevity, we shall frame our arguments as though each curve $X \in \mathcal{E}(P)$ is of the form

$$
\left\{\left(z_{1}, z_{2}\right): z_{1}^{m_{1} / \operatorname{gcd}\left(m_{1}, m_{2}\right)}=\xi z_{2}^{m_{2} / \operatorname{gcd}\left(m_{1}, m_{2}\right)}\right\}
$$


for some $\xi \in \mathbb{C}$, and the reasons are the same as for Proposition 5.3 (although see the remark following (6.13) ). It would be useful to look at the fourth assertion above in greater depth. First observe that

$$
\frac{m_{1}}{\operatorname{gcd}\left(m_{1}, m_{2}\right)}=\sigma_{2}, \quad \frac{m_{2}}{\operatorname{gcd}\left(m_{1}, m_{2}\right)}=\sigma_{1},
$$

and hence note that for any $\xi \in \mathbb{C}$ such that $\left\{\left(z_{1}, z_{2}\right): z_{1}^{\sigma_{2}}=\xi z_{2}^{\sigma_{1}}\right\} \in \mathcal{E}(P)$, $\Pi$ is forced to be harmonic along each of the complex lines that make up the set

$$
\mathbf{L}(\xi):=\bigcup_{l=0}^{\sigma_{1} \sigma_{2}-1}\left\{\left(t_{1}, t_{2}\right): t_{1}=|\xi|^{1 / \sigma_{1} \sigma_{2}} \exp \left(\frac{2 \pi i l+i \operatorname{Arg}(\xi)}{\sigma_{1} \sigma_{2}}\right) t_{2}\right\}
$$

(here Arg denotes some branch of the argument). But since Proposition 5.3 is applicable to the domain $\omega_{R}$, there must be only finitely many complex lines of the above description. This implies Part (1) of our theorem.

Let us introduce some notation that we shall require. By Part (1), and in view of the italicised remark above, let us label the irreducible curves belonging to $\mathcal{E}(P)$ as $X_{1}, \ldots, X_{N}$, and let us assume that

$$
X_{j}=\left\{\left(z_{1}, z_{2}\right): z_{1}^{m_{1} / \operatorname{gcd}\left(m_{1}, m_{2}\right)}=\xi_{j} z_{2}^{m_{2} / \operatorname{gcd}\left(m_{1}, m_{2}\right)}\right\}
$$

for some $\xi_{j} \in \mathbb{C}, j=1, \ldots, N$. For each $j \leq N$, let us denote the collection of complex lines in the set $\mathbf{L}\left(\xi_{j}\right)$, as defined above, by $\left\{L_{j 0}, L_{j 1}, \ldots, L_{j\left(\sigma_{1} \sigma_{2}-1\right)}\right\}$. In keeping with this numbering scheme, let $\mu_{j k} \in \mathbb{Z}_{+}$denote the numbers determined by (5.1) for the pair $(\Pi, R)$. Similarly, let $M_{j k} \in \mathbb{Z}_{+}$be the integer associated to the complex line $L_{j k} \in \mathcal{E}(\Pi)$ that is provided by the Proposition [5.3. Let $\Delta:=$ $\max \left\{2 M_{j k}: 1 \leq j \leq N, 0 \leq k \leq \sigma_{1} \sigma_{2}-1\right\}$. With these notations, we are ready to construct the desired functions.

Step 1. Constructing $G$ and the auxiliary functions

Consider the unitary transformations $R^{l m}:\left(z_{1}, z_{2}\right) \longmapsto\left(e^{2 \pi i l / \sigma_{1}} t_{1}, e^{2 \pi i m / \sigma_{2}} t_{2}\right)$, $l, m \in \mathbb{Z}$. Recall that if $\rho$ is as given by Proposition 5.3 when applied to the domain $\omega_{R}$, then $\rho$ is obtained by subtracting from $R$ all pluriharmonic terms of degree $\leq \Delta$ occurring in the Taylor expansion of $R$ around $u=0$. By construction

$$
\begin{aligned}
\rho \circ R^{l m}(t) & =\rho(t) \quad \forall t \in \mathbb{C}^{2}, \quad \forall l, m \in \mathbb{Z}, \\
\Pi \circ R^{l m}(t) & =\Pi(t) \quad \forall t \in \mathbb{C}^{2}, \quad \forall l, m \in \mathbb{Z} .
\end{aligned}
$$

From the transformation properties of the Levi form, we conclude from (6.2) that

$$
\mathfrak{L} \Pi\left(R^{l m} t ; R^{l m} V\right)=\mathfrak{L} \Pi(t ; V) \quad \forall(t, V) \in \mathbb{C}^{2} \times \mathbb{C}^{2}, \quad \forall l, m \in \mathbb{Z} .
$$

Hence, if we define $\mathfrak{N}_{\Pi}(t)$ to be the null-space of $\mathfrak{L} \Pi(t ; \cdot)$, then the above statement reveals that:

(i) With $\omega(\Pi)$ as defined in Section 2, $t \in \omega(\Pi)$ and $V \in \mathfrak{N}_{\Pi}(t)$ if and only if $R^{l m}(t) \in \omega(\Pi)$ and $R^{l m}(V) \in \mathfrak{N}_{\Pi}\left(R^{l m}(t)\right), l, m \in \mathbb{Z}$.

If, for each triple $(k, l, m)$ with $k, l, m$ belonging to the respective integer-ranges established above, we define $\varkappa(k, l, m)$ by the relation

$$
0 \leq \varkappa(l, m, n) \leq \sigma_{1} \sigma_{2}-1 \text { and } \varkappa(l, m, n) \equiv\left(k+\sigma_{2} l-\sigma_{1} m\right) \bmod \left(\sigma_{1} \sigma 2\right),
$$

then

Now note that since $\operatorname{gcd}\left(\sigma_{1}, \sigma_{2}\right)=1$,

$$
R^{l m}\left(L_{j k}\right)=L_{j, \varkappa(k, l, m)} .
$$

For each $k=0, \ldots, \sigma_{1} \sigma_{2}-1, \exists l, m \in \mathbb{Z}$ such that $k=\sigma_{2} l-\sigma_{1} m$. 
From the observation $(i)$, the prescription provided by Proposition 4.2 for constructing each $H_{j k}$ associated to a complex line $L_{j k}$, and from (6.3), it is clear that in the construction of the $\widetilde{G}_{\delta}$ given by the equation (5.9):

(ii) For each $k$, we may set $\overline{\mathcal{K}}_{j k}^{n}=R^{l m}\left(\overline{\mathcal{K}}_{j 0}^{n}\right), n=1,2$, taking appropriate $l, m \in \mathbb{Z}$

(iii) For each $k$, we may set $H_{j k}=H_{j 0} \circ\left(R^{l m}\right)^{-1}$ and $\Psi_{j k}=\Psi_{j 0} \circ\left(R^{l m}\right)^{-1}$, taking appropriate $l, m \in \mathbb{Z}$;

and the conclusions of Proposition 5.3 will continue to hold true. That we can always find appropriate $l, m \in \mathbb{Z}$ for the purposes of $(i i)$ and (iii) follows from (6.4).

Result 5.1 is applicable to $\Pi$. A careful examination of its proof reveals that Noell's construction of the bumping is local. Hence, in view of (6.2) and (i), we can construct the function $H_{0}$ that we use in the proof of Proposition 5.3, as well as the perturbation described in (A) and (B) in the proof of Proposition 5.3 in such a way that:

$$
H \circ R^{l m}(t)=H(t) \forall u \in \mathbb{C}^{2} \text { and } \forall l, m \in \mathbb{Z} .
$$

And finally, owing to (6.1):

$$
\begin{aligned}
M_{j k} & =M_{j k^{*}}=: M_{j} \text { for any } k \neq k^{*}, \\
u_{j k}(x) & =\Pi_{2 M_{j}}\left(\left|\xi_{j}\right|^{1 / \sigma_{1} \sigma_{2}} \exp \left(\frac{2 \pi i k+i \operatorname{Arg}\left(\xi_{j}\right)}{\sigma_{1} \sigma_{2}}\right) x, x\right), \quad \forall x \in \mathbb{C},
\end{aligned}
$$

where $k=0, \ldots, \sigma_{1} \sigma_{2}-1$.

Recall that the change of coordinate mentioned in Proposition 5.3 is represented by a biholomorphism of the form

$$
\tau_{1}\left(s, t_{1}, t_{2}\right):=\left(s-q\left(t_{1}, t_{2}\right), t_{1}, t_{2}\right), \quad\left(s, t_{1}, t_{2}\right) \in \mathbb{C}^{3},
$$

where $q$ is the holomorphic polynomial that is the sum of all monomials in $t_{1}$ and $t_{2}$ whose real and imaginary parts are the pluriharmonic terms of some $\Pi_{n, R}, n=$ $\nu+1, \ldots, \Delta:=\max _{j, k} 2 M_{j k}$. However, since $R=\varphi \circ \Psi$, every monomial in $q$ is the product of integer powers of $t_{1}^{\sigma_{1}}$ and $t_{2}^{\sigma^{2}}$ (in fact, this observation is the implicit reason for the the assertion (6.1) above). Thus, a diagram-chase reveals that there is a biholomorphism $\tau_{2}: \mathbb{C}^{3} \longrightarrow \mathbb{C}^{3}$ that makes the following diagram commute:

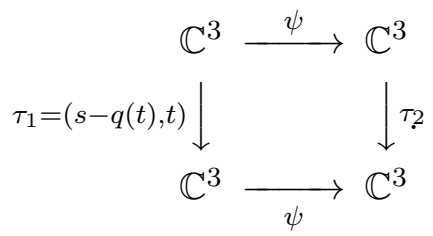

Furthermore $\tau_{2}$ is of the form

$$
\tau_{2}\left(w, z_{1}, z_{2}\right)=\left(w-f\left(z_{1}, z_{2}\right), z_{1}, z_{2}\right),\left(w, z_{1}, z_{2}\right) \in \mathbb{C}^{3},
$$

where $f$ is a polynomial in $z_{1}$ and $z_{2}$. Let us write $\left(\widehat{w}, z_{1}, z_{2}\right):=\tau_{2}\left(w, z_{1}, z_{2}\right)$, and let

$$
\Omega \bigcap U_{\zeta}=\{(\widehat{w}, z): \mathfrak{R e} \widehat{w}+\widetilde{\varrho}(z)+\widetilde{r}(\mathfrak{I m} \widehat{w}, z)<0\}
$$

denote the local representation of $\Omega$ relative to the new coordinate system $\left(U_{\zeta} ; \widehat{w}, z_{1}, z_{2}\right)$. Then, it is clear that

$$
\psi \circ \tau_{1}\left(\omega_{R}\right)=\left\{(\widehat{w}, z) \in \tau_{2}\left(U_{\zeta}\right): \mathfrak{R e} \widehat{w}+\widetilde{\varrho}(z)<0\right\} .
$$


We now pick and fix a value in $\left(0, \delta_{0}\right)$, say $\delta_{0} / 2$, and use this to define the functions mentioned in the statement of our theorem. To this end, we define:

$$
\begin{aligned}
& G(z):= \frac{1}{\sigma_{1} \sigma_{2}} \sum_{a=1}^{\sigma_{1}} \sum_{b=1}^{\sigma_{2}} G_{\delta_{0} / 2}\left(\left|z_{1}\right|^{1 / \sigma_{1}} \exp \left(\frac{2 \pi i a+i \operatorname{Arg}\left(z_{1}\right)}{\sigma_{1}}\right),\right. \\
&\left.\left|z_{2}\right|^{1 / \sigma_{2}} \exp \left(\frac{2 \pi i b+i \operatorname{Arg}\left(z_{2}\right)}{\sigma_{2}}\right)\right) \\
& \overline{\mathcal{W}}_{j}^{l}:=\Psi\left(\overline{\mathcal{K}}_{j 0}^{l}\right), \quad l=1,2, \quad j=1, \ldots, N
\end{aligned}
$$

where all the objects on the right-hand sides of (6.9) and (6.10) are as given in Proposition 5.3. We remark here that, owing to $(i i)$ above, we could as well have used $\overline{\mathcal{K}}_{j k}^{l}, k=0, \ldots, \sigma_{1} \sigma_{2}-1$, in the definition (6.10) for each fixed $j$. Let us now define

$$
\begin{gathered}
\mathcal{H}_{0}(z):=\frac{\delta_{0}}{2 \sigma_{1} \sigma_{2}} \sum_{a=1}^{\sigma_{1}} \sum_{b=1}^{\sigma_{2}} H\left(\left|z_{1}\right|^{1 / \sigma_{1}} \exp \left(\frac{2 \pi i a+i \operatorname{Arg}\left(z_{1}\right)}{\sigma_{1}}\right),\right. \\
\left.\left|z_{2}\right|^{1 / \sigma_{2}} \exp \left(\frac{2 \pi i b+i \operatorname{Arg}\left(z_{2}\right)}{\sigma_{2}}\right)\right) .
\end{gathered}
$$

As $H$ is homogeneous of degree $\nu, \mathcal{H}_{0}$ is $\left(m_{1}, m_{2}\right)$-homogeneous. The other properties of $\mathcal{H}_{0}$ listed in Theorem 2.5 are immediate. Furthermore, note that:

$$
\begin{gathered}
P(z)=\frac{1}{\sigma_{1} \sigma_{2}} \sum_{a=1}^{\sigma_{1}} \sum_{b=1}^{\sigma_{2}} \Pi\left(\left|z_{1}\right|^{1 / \sigma_{1}} \exp \left(\frac{2 \pi i a+i \operatorname{Arg}\left(z_{1}\right)}{\sigma_{1}}\right),\right. \\
\left.\left|z_{2}\right|^{1 / \sigma_{2}} \exp \left(\frac{2 \pi i b+i \operatorname{Arg}\left(z_{2}\right)}{\sigma_{2}}\right)\right) .
\end{gathered}
$$

Comparing the equations (6.9) and (6.11) with our definition of $\mathcal{H}_{0}$, and keeping (6.10) in mind, we already have the first and the third properties listed at the end of Theorem 2.5. The task of defining the $v_{j}$ 's is subtler. If we would like to recover the second property listed at the end of Theorem [2.5, we would first need to study the quantity $\left.\left(G-P+\mathcal{H}_{0}\right)\right|_{\overline{\mathcal{W}}_{j}^{1}}$. Accordingly, we first fix a $J \leq N$, then refer to the formula for $\widetilde{\Gamma}_{\delta_{0} / 2}$ from (5.14), whence for an $R>0$ sufficiently small, we get:

$$
\begin{gathered}
\left.\left(G-P+\mathcal{H}_{0}\right)\right|_{\overline{\mathcal{W}}_{J}^{1}} \\
=\frac{1}{\sigma_{1} \sigma_{2}} \sum_{k=0}^{\sigma_{1} \sigma_{2}-1} \sum_{a=1}^{\sigma_{1}} \sum_{b=1}^{\sigma_{2}} \Psi_{J k}\left(\left|z_{1}\right|^{1 / \sigma_{1}} \exp \left(\frac{2 \pi i a+i \operatorname{Arg}\left(z_{1}\right)}{\sigma_{1}}\right)\right. \\
\left.\left|z_{2}\right|^{1 / \sigma_{2}} \exp \left(\frac{2 \pi i b+i \operatorname{Arg}\left(z_{2}\right)}{\sigma_{2}}\right)\right) U_{J k, \delta_{0} / 2}\left(\left|z_{2}\right|^{1 / \sigma_{2}} \exp \left(\frac{2 \pi i b+i \operatorname{Arg}\left(z_{2}\right)}{\sigma_{2}}\right)\right) \\
\forall z \in \overline{\mathcal{W}}_{J}^{1} \bigcap \mathbb{B}^{2}(0 ; R) .
\end{gathered}
$$

We refer the reader to the proof of Proposition 5.3. and recall the numbering scheme introduced above, for the meaning of the quantities $U_{j k, \delta_{0} / 2}$ in (6.12). Following (5.14) strictly, there should be a double-sum over all possible values of $(j, k) \in$ $\{1, \ldots, N\} \times\left\{0, \ldots, \sigma_{1} \sigma_{2}-1\right\}$. It is easy to see, however, that for $z \in \overline{\mathcal{W}}_{J}^{1}$, the summands involving the indices $j \neq J$ vanish. Note the following points that follow by construction: 
- Since, by definition, for each $j=1, \ldots, N, \Pi_{2 M_{j}}$ is the sum of monomials of degree $2 M_{j}$ that are products of integer powers of $t_{l}^{\sigma_{l}}$ and $\bar{t}_{l}^{\sigma_{l}}, l=1,2$, the equation (6.6) tells us that the second factor in the expression (6.12) does not change as $b$ ranges through the set $\left\{1, \ldots, \sigma_{2}\right\}$.

- In view of $(i i),(i i i)$ and (6.10), the first factor in (6.12) does not change as $k$ ranges through the set $\left\{0, \ldots, \sigma_{1} \sigma_{2}-1\right\}$.

In view of these two points, the equation (6.12) reveals that the second property listed at the end of Theorem 2.5 is established if we define

$$
v_{j}(x):=\frac{1}{\sigma_{1} \sigma_{2}} \sum_{k=0}^{\sigma_{1} \sigma_{2}-1}\left[\sum_{b=1}^{\sigma_{2}} U_{j k, \delta_{0} / 2}\left(|x|^{1 / \sigma_{2}} \exp \left(\frac{2 \pi i b+i \operatorname{Arg}(x)}{\sigma_{2}}\right)\right)\right] \forall x \in \mathbb{C},
$$

for each $j=1, \ldots, N$. The second property is established because, for a fixed $J \leq N$ and $z \in \overline{\mathcal{W}}_{J}^{1}$, the right-hand side of the above equation (with $x$ replaced by $z_{2}$ ) is just a different way of expressing the right-hand side of (6.12). We claim that each $v_{j}$ is subharmonic. To see this, we note that the functions within the square brackets in (6.13) are subharmonic. This follows from the following general fact:

Fact 6.2. Let $D_{1}$ and $D_{2}$ be two domains in $\mathbb{C}^{n}$ and let $p: D_{1} \longrightarrow D_{2}$ be a proper holomorphic map. If $u$ is a plurisubharmonic function on $D_{1}$, then the function

$$
v(z):=\sum_{t \in p^{-1}\{z\}} u(t), \quad z \in D_{2}
$$

is plurisubharmonic on $D_{2}$.

Therefore $v_{j}$, being a positive linear combination of subharmonic functions, is subharmonic. In fact, as each $U_{j k, \delta}$ is, by construction, strictly subharmonic away from $x=0$, and since the map $x \longmapsto x^{\sigma_{2}}$ is a local biholomorphism away fron $x=0$, each $v_{j}$ is, in fact, strictly subharmonic on $\mathbb{C} \backslash\{0\}$. Note that if for some $j \leq N$, say $j^{0}, X_{j^{0}}$ is the $z_{1}$-axis, then — in view of the work done in Proposition 5.3 there would be just a single $U_{j^{0} k, \delta_{0} / 2}=: U_{j^{0}, \delta_{0} / 2}$. Accordingly, the formulas (6.9) and (6.13) would reflect the following changes:

- In defining $v_{j^{0}}$, the $U_{j k, \delta_{0} / 2}$ 's in (6.13) would be replaced by $U_{j^{0}, \delta_{0} / 2}$ and every occurrance of $\sigma_{2}$ would be replaced by $\sigma_{1}$.

- In (6.9), $v_{j}$ would appear as a term depending on $z_{1}$.

Since $G_{\delta_{0} / 2}$ is, by construction, plurisubharmonic on $\mathbb{C}^{2}$, we see from Fact 6.2 , and the formula (6.9), that $G$ is plurisubharmonic on $\mathbb{C}^{2}$. At this stage, we only need to establish (2.1). This will require one more holomorphic change of coordinate.

Step 2. Establishing the relation (2.1)

Let $R_{1}>0$ be so small that $\mathbb{B}^{3}\left(0 ; R_{1}\right) \subset V_{\zeta}$. Shrinking $R_{1}$ further if necessary, it follows from our definition (6.10) of the $\left(m_{1}, m_{2}\right)$-wedges $\overline{\mathfrak{W}}_{j}^{1}, j=1, \ldots, N$, and from Part 2e) of Proposition 5.3 (applied to the domain $\omega_{R}$ ) that there is a constant $A_{1}>0$ such that

$$
(G-\widetilde{\varrho})(z) \leq-A_{1}\left(\left|z_{1}\right|^{m_{1}}+\left|z_{2}\right|^{m_{2}}\right) \quad \forall\left(z_{1}, z_{2}\right) \in\left(\mathbb{C}^{2} \backslash \cup_{j=1}^{N} \overline{\mathcal{W}}_{j}^{1}\right) \bigcap \mathbb{B}^{2}\left(0 ; R_{1}\right) .
$$


Recall that $\widetilde{\varrho}$ is as given by (6.8). Let us now fix a $j \leq N$. Now, obviously:

$$
\begin{aligned}
&(G-\widetilde{\varrho})(z):= \frac{1}{\sigma_{1} \sigma_{2}} \sum_{a=1}^{\sigma_{1}} \sum_{b=1}^{\sigma_{2}}\left(G_{\delta_{0} / 2}-\rho\right)\left(\left|z_{1}\right|^{1 / \sigma_{1}} \exp \left(\frac{2 \pi i a+i \operatorname{Arg}\left(z_{1}\right)}{\sigma_{1}}\right)\right. \\
&\left.\left|z_{2}\right|^{1 / \sigma_{2}} \exp \left(\frac{2 \pi i b+i \operatorname{Arg}\left(z_{2}\right)}{\sigma_{2}}\right)\right) \\
& \equiv \frac{1}{\sigma_{1} \sigma_{2}} \sum_{a=1}^{\sigma_{1}} \sum_{b=1}^{\sigma_{2}} Y(z ; a, b)
\end{aligned}
$$

Note that if $z \in \overline{\mathcal{W}}_{j}^{1}$, then, from our discussions above, the arguments of the functions constituting the sum above would fall into one of the cones $\overline{\mathcal{K}}_{j k}^{1}, k=$ $0, \ldots, \sigma_{1} \sigma_{2}-1$. Applying Part 2d) of Proposition [5.3, we get

$$
\begin{aligned}
z & \in \overline{\mathcal{W}}_{j}^{1} \text { is such that }\left(\left|z_{1}\right|^{1 / \sigma_{1}} e^{i \frac{2 \pi a+\operatorname{Arg}\left(z_{1}\right)}{\sigma_{1}}},\left|z_{2}\right|^{1 / \sigma_{2}} e^{i \frac{2 \pi b+\operatorname{Arg}\left(z_{2}\right)}{\sigma_{2}}}\right) \text { lies } \\
\text { in } & \overline{\mathcal{K}}_{j k}^{1} \bigcap \mathbb{B}^{2}\left(0 ; r_{\delta_{0} / 2}\right) \\
& \Longrightarrow Y(z ; a, b) \\
& \leq-\frac{C \delta_{0}}{2}\left\{\left.|| z_{1}\right|^{1 / \sigma_{1}} e^{i \frac{2 \pi a+\operatorname{Arg}\left(z_{1}\right)}{\sigma_{1}}}-\left.\xi_{j k}\left|z_{2}\right|^{1 / \sigma_{2}} e^{i \frac{2 \pi b+\operatorname{Arg}\left(z_{2}\right)}{\sigma_{2}}}\right|^{\mu_{j}}\left|z_{2}\right|^{\frac{\nu-\mu_{j}}{\sigma_{2}}}+\left|z_{2}\right|^{\frac{2 M_{j}}{\sigma_{2}}}\right\},
\end{aligned}
$$

where $\xi_{j k}:=\left|\xi_{j}\right|^{1 / \sigma_{1} \sigma_{2}} \exp \left(i \frac{2 \pi k+\operatorname{Arg}\left(\xi_{j}\right)}{\sigma_{1} \sigma_{2}}\right)$, and where we remind the reader that $\mu_{j 0}=\cdots=\mu_{j\left(\sigma_{1} \sigma_{2}-1\right)}=: \mu_{j}$ and $M_{j 0}=\cdots=M_{j\left(\sigma_{1} \sigma_{2}-1\right)}=: M_{j}$. Thus, if $R_{2}>0$ is so small that the the arguments of the functions constituting the right-hand side of (6.15) are in $\mathbb{B}^{2}\left(0 ; r_{\delta_{0} / 2}\right)$, then, from (6.15) and (6.16), we have a non-negative function $\mathcal{N}_{j}: \mathbb{B}^{2}\left(0 ; R_{2}\right) \longrightarrow[0, \infty)$ such that

$$
(G-\widetilde{\varrho})(z) \leq-\frac{C \delta_{0}}{2} \mathcal{N}_{j}(z) \quad \forall\left(z_{1}, z_{2}\right) \in \overline{\mathcal{W}}_{j}^{1} \bigcap \mathbb{B}^{2}\left(0 ; R_{2}\right) .
$$

Now, the whole point of the estimate in $2 d$ ) (applied to the domain $\omega_{R}$ ) above was that there exists a constant $A_{2}>0$ such that

$$
|\rho(t)| \leq A_{2}\left(\left|t_{1}-\xi_{j k} t_{2}\right|^{\mu_{j}}\left|t_{2}\right|^{\nu-\mu_{j}}+\left|t_{2}\right|^{2 M_{j}}\right) \quad \forall t \in \overline{\mathcal{K}}_{j k}^{1} \bigcap \operatorname{Dom}(\rho),
$$

for all relevant $(j, k)$. Hence, if $z \in \overline{\mathcal{W}}_{j}^{1}$ satisfies the hypothesis of the statement (6.16), then $|\widetilde{\varrho}(z)|$ has as upper bound the quantity obtained from the right-hand side of (6.16) with the factor $-\left(C \delta_{0} / 2\right)$ replaced by $A_{2}$. Eventually, therefore, we get the estimate

$$
|\widetilde{\varrho}(z)| \leq A_{2} \mathcal{N}_{j}(z) \quad \forall\left(z_{1}, z_{2}\right) \in \overline{\mathcal{W}}_{j}^{1} \bigcap \mathbb{B}^{2}\left(0 ; R_{2}\right) .
$$

We are now ready to establish (2.1). To this end, we define new coordinates $\left(W, Z_{1}, Z_{2}\right)$ as follows

$$
W:=\widehat{w}+K \widehat{w}^{2}, \quad Z_{l}:=z_{l}, \quad l=1,2,
$$

where $K>0$. The mapping $(\widehat{w}, z) \longmapsto(W, Z)$ is invertible in a neighbourhood of $0 \in \mathbb{C}^{3}$, whose size depends on the parameter $K$. We will choose a suitable $K>0$ so that (2.1) is achieved. Recall, from (6.8), the relation $\widetilde{r}(\mathfrak{I m} \widehat{w}, z)=r(\mathfrak{I m} w, z)$. 
Let us write $\mathfrak{R e} \widehat{w}=: \alpha$ and $\mathfrak{I m} \widehat{w}=: \beta$. In view of $(* *)$, there exists an $A_{3}>0$ such that

$$
\begin{aligned}
|\widetilde{r}(\beta, z)|=|r(\mathfrak{I m} w, z)| & \leq A_{3}|\mathfrak{I m}(\widehat{w}+f(z))|^{q} \\
& \leq A_{3}\left(|\beta|^{q}+|\mathfrak{I m}(f(z))|^{q}\right) \quad \forall(\widehat{w}, z) \in \overline{\mathbb{B}^{3}\left(0 ; R_{1} / 2\right)}
\end{aligned}
$$

where $q:=\Delta_{1}(\partial \Omega)+(1 / \nu)$ by hypothesis, and where $f$ is the polynomial described in (6.7). Note that if we decompose $f$ as

$$
f=\sum_{\eta} \mathcal{Q}_{\eta, f},
$$

where the right-hand side is as described in the statement of Fact 6.1, and if each $\mathcal{Q}_{\eta, f} \circ \Psi$ is the sum of pluriharmonic terms contained in some $\Pi_{n, R}$, then $\eta \geq \nu+1$. This is because $\Pi_{\nu, R}$ itself contains no pluriharmonic terms. Then, in view of Fact 6.1 and (6.19), and raising the value of $A_{3}>0$ if necessary, we have

$$
|\widetilde{r}(\beta, z)| \leq A_{3}\left(|\beta|^{q}+\left(\left|z_{1}\right|^{m_{1}}+\left|z_{2}\right|^{m_{2}}\right)^{q(\nu+1) / \nu}\right) \quad \forall z \in \overline{\mathbb{B}^{2}\left(0 ; R_{1} / 2\right)} .
$$

By a similar appeal to Fact 6.1 (and raising $A_{3}>0$ even further if necessary):

$$
\mathcal{N}_{j}(z) \geq A_{3}^{-1}\left(\left|z_{1}\right|^{m_{1}}+\left|z_{2}\right|^{m_{2}}\right)^{(\Delta+1) / \nu} \forall\left(z_{1}, z_{2}\right) \in \overline{\mathcal{W}}_{j}^{1} \bigcap \mathbb{B}^{2}\left(0 ; R_{2}\right),
$$

for each $j=1, \ldots, N$. Recall that $\Delta:=\max \left\{2 M_{j k}: j \leq N, k=0, \ldots, \sigma_{1} \sigma_{2}-1\right\}$.

Note that we would be done if we could show that:

For each $(\widehat{w}, z) \neq 0$ such that $(\alpha+\widetilde{\varrho}(z)+\widetilde{r}(\beta, z))=0$, provided $|\widehat{w}|$ and $\|z\|$

are sufficiently small, $\left(\mathfrak{R e}\left(\widehat{w}+K \widehat{w}^{2}\right)+G(z)\right)<0$.

The above statement is true when $z \in\left(\left(\mathbb{C}^{2} \backslash \cup_{j=1}^{N} \overline{\mathcal{W}}_{j}^{1}\right) \bigcap \mathbb{B}^{2}\left(0 ; R_{1}\right)\right)$, for $R_{1}>0$ sufficiently small, and for a suitable choice of $K>0$. This follows from the estimate (6.14); it was shown by Yu [14, pp. 604-605] using an idea of Fornaess-Sibony [10].

It thus remains to establish the above statement when $z \in \overline{\mathcal{W}}_{j}^{1}$ (provided $(\widehat{w}, z) \neq$ 0 and sufficiently close to the origin). We fix $j \leq N$ for the following calculation and write

$$
\begin{aligned}
\mathfrak{R e}\left(\widehat{w}+K \widehat{w}^{2}\right)+G(z)= & \alpha+K\left(\alpha^{2}-\beta^{2}\right)+G(z) \\
= & -K \beta^{2}-(\widetilde{\varrho}(z)+\widetilde{r}(\beta, z))+K(\widetilde{\varrho}(z)+\widetilde{r}(\beta, z))^{2}+G(z) \\
\leq & -K \beta^{2}+(G-\widetilde{\varrho})(z)+|\widetilde{r}(\beta, z)|+2 K\left(\widetilde{\varrho}(z)^{2}+\widetilde{r}(\beta, z)^{2}\right) \\
& \quad \text { provided } \alpha+\widetilde{\varrho}(z)+\widetilde{r}(\beta, z)=0,(\widehat{w}, z) \neq 0 . \quad
\end{aligned}
$$

Let us write $R:=\min \left(R_{1} / 2, R_{2}\right)$. Then, applying to (6.22) the estimates (6.17), (6.18) and (6.20), and shrinking $R>0$ if necessary so that $2 K \widetilde{r}(\beta, z)^{2} \leq\left(K / 2 A_{3}\right)|\widetilde{r}(\beta, z)|$, we get

$$
\begin{aligned}
& \mathfrak{R e}\left(\widehat{w}+K \widehat{w}^{2}\right)+G(z) \\
& \leq-K \beta^{2}+\left[2 K A_{2}^{2} \mathcal{N}_{j}^{2}(z)-\frac{C \delta_{0}}{2} \mathcal{N}_{j}(z)\right]+\left(1+\left(K / 2 A_{3}\right)\right)|\widetilde{r}(\beta, z)| \\
& \leq\left(-\frac{K}{2}+A_{3}\right) \beta^{2}+\left[2 K A_{2}^{2} \mathcal{N}_{j}^{2}(z)-\frac{C \delta_{0}}{2} \mathcal{N}_{j}(z)\right] \\
& +\left(A_{3}+\frac{1}{2} K\right)\left(\left|z_{1}\right|^{m_{1}}+\left|z_{2}\right|^{m_{2}}\right)^{q(\nu+1) / \nu} \quad \forall z \in \overline{\mathcal{W}}_{j}^{1} \bigcap\left(\mathbb{B}^{3}(0 ; R) \backslash\{0\}\right) .
\end{aligned}
$$


At this stage, we fix $K>0$ once and for all so that $\left(-(K / 2)+A_{3}\right) \leq-1$ and is large enough for Yu's argument; this determines the final holomorphic coordinates required for our theorem. With $K$ fixed, we can shrink $R>0$ further if necessary and apply (6.21) so that the following inequalities follow (let us write $A:=A_{3}+\frac{1}{2} K$, $B:=C \delta_{0} / 4$ and $\left.\Sigma(z):=\left|z_{1}\right|^{m_{1}}+\left|z_{2}\right|^{m_{2}}\right)$ :

$$
\begin{gathered}
\mathfrak{R e}\left(\widehat{w}+K \widehat{w}^{2}\right)+G(z) \leq-\beta^{2}-B \mathcal{N}_{j}(z)+A\left(\left|z_{1}\right|^{m_{1}}+\left|z_{2}\right|^{m_{2}}\right)^{q(\nu+1) / \nu} \\
\leq-\beta^{2}-\left(B / A_{3}\right) \Sigma(z)^{(\Delta+1) / \nu}+A \Sigma(z)^{q(\nu+1) / \nu} \\
\forall z \in \overline{\mathcal{W}}_{j}^{1} \bigcap\left(\mathbb{B}^{3}(0 ; R) \backslash\{0\}\right) .
\end{gathered}
$$

The curves $X_{j}$ have order of contact $2 M_{j} / \nu$ with $\partial \Omega$ at the origin. Hence $\Delta_{1}(\partial \Omega) \geq$ $\Delta / \nu$. This means

$$
\left(\Delta_{1}(\partial \Omega)+\frac{1}{\nu}\right) \frac{\nu+1}{\nu}>\frac{\nu \Delta_{1}(\partial \Omega)+1}{\nu} \geq \frac{\Delta+1}{\nu} .
$$

Applying this to (6.24) affords us an $R>0$ sufficiently small that

$\mathfrak{R e}\left(\widehat{w}+K \widehat{w}^{2}\right)+G(z) \leq-\beta^{2}-\frac{B}{2 A_{3}} \Sigma(z)^{(\Delta+1) / \nu}<0 \forall z \in \overline{\mathcal{W}}_{j}^{1} \bigcap\left(\mathbb{B}^{3}(0 ; R) \backslash\{0\}\right)$,

for each $j=0, \ldots, N$. In view of our preceding remarks, the proof is complete.

\section{REFERENCES}

[1] E. Bedford and J.E. Fornaess, A construction of peak functions on weakly pseudoconvex domains, Ann. of Math. 107 (1978), 555-568.

[2] G. Bharali and B. Stensønes, Plurisubharmonic polynomial polynomials and bumping, Math. Z. 261 (2009), 39-63.

[3] D. Catlin, Boundary invariants of pseudoconvex domains, Ann. of Math.(2) 120 (1984), 529586.

[4] B.-Y. Chen, J. Kamimoto and T. Ohsawa, Behavior of the Bergman kernel at infinity, Math. Z. 248 (2004), 695-708.

[5] J.P. D’Angelo, Real hypersurfaces, orders of contact, and applications, Annals of Math. 115 (1982), 615-637.

[6] K. Diederich and J.E. Fornaess, Pseudoconvex domains: bounded strictly plurisubharmonic exhaustion functions, Invent. Math. 39 (1977), 129-141.

[7] K. Diederich and J.E. Fornaess, Proper holomorphic maps onto pseudoconvex domains with real-analytic boundary, Ann. of Math. 110 (1979), 575-592.

[8] K. Diederich and G. Herbort, Pseudoconvex domains of semiregular type, Contributions to Complex Analysis and Analytic Geometry, Aspects Math. (1994), Vieweg, Braunschweig, pp. $127-161$

[9] J.E. Fornaess, Sup-norm estimates for $\bar{\partial}$ in $\mathbb{C}^{2}$, Ann. of Math. 123 (1986), 335-345.

[10] J.E. Fornaess and N. Sibony, Construction of p.s.h. functions on weakly pseudoconvex domains, Duke Math. J. 58 (1989), 633-655.

[11] A. Noell, Peak functions for pseudoconvex domains, Several Complex Variables - Proceedings of the Mittag-Leffler Institute, 1987-1988, Princeton University Press, Princeton, NJ, 1993, pp. 529-541.

[12] R.M. Range, Integral kernels and Hölder estimates for $\bar{\partial}$ on pseudoconvex domains of finite type in $\mathbb{C}^{2}$, Math. Ann. 288 (1990), 63-74.

[13] J.Y. Yu, Peak functions on weakly pseudoconvex domains, Indiana Univ. Math. J. 43 (1994), 1271-1295.

[14] J. Yu, Weighted boundary limits of the generalized Kobayashi-Royden metrics on weakly pseudoconvex domains, Trans. Amer. Math. Soc. 347 (1995), 587-613.

Department of Mathematics, Indian Institute of Science, Bangalore - 560012

E-mail address: bharali@math.iisc.ernet.in 